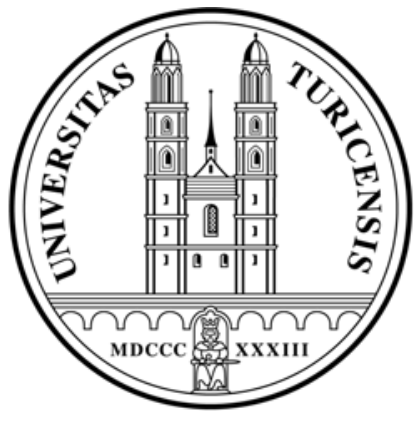

Institute for Empirical Research in Economics

University of Zurich

Working Paper Series

ISSN 1424-0459

Working Paper No. 237

An Adverse Selection Model of Optimal Unemployment Insurance

Marcus Hagedorn, Ashok Kaul and Tim Mennel

March 2005 


\title{
An Adverse Selection Model of Optimal Unemployment Insurance
}

\author{
Marcus Hagedorn*, Ashok Kaul ${ }^{\dagger}$ and Tim Mennel ${ }^{\ddagger}$
}

First draft: April 2002. This version: October 2004

\begin{abstract}
We ask whether offering a menu of unemployment insurance contracts is welfare improving in a heterogeneous population. We adopt a repeated moral-hazard framework as in Shavell/Weiss (1979) supplemented by unobserved heterogeneity about agents' job opportunities. Our main theoretical contribution is an analytical characterization of the sets of jointly feasible entitlements that renders an efficient computation of these sets feasible. Our main economic result is that optimal contracts for "bad" searchers tend to be upward-sloping due to an adverse-selection effect. This is in contrast to the well-known optimal decreasing time-profile of benefits in pure moral hazard environments that continue to be optimal for "good" searchers in our model.
\end{abstract}

Keywords: Unemployment Insurance, Recursive Contracts, Adverse Selection, Repeated Moral Hazard

JEL classification: J65, J64, D82, C61, E61

\footnotetext{
*Hagedorn: University of Bonn, Mailing address: University of Pennsylvania, Department of Economics, 3718 Locust Walk, Philadelphia, 19104 PA, email: hagedorn@econ.upenn.edu, Germany.

${ }^{\dagger}$ Kaul: Universitat Pompeu Fabra, Ramon Trias Fargas 25-27, 08005 Barcelona, Spain; ashok.kaul@upf.edu and IZA, University of Bonn.

${ }^{\ddagger}$ Mennel (Correspondence), Center for European Integration Studies (ZEIb), University of Bonn, Walter-Flex-Str. 3, D-53115 Bonn, tmennel@wiwi.uni-bonn.de.
} 


\section{Introduction}

People flowing into unemployment are heterogeneous with respect to their chances of finding a new job. These differences include different innate ability, general education, human capital accumulated on the the last job and their unemployment history. Also, chances of reemployment generally vary over different segments of the labor market. Typically, workers searching for a job are better informed about their job opportunities than a government agency in charge of providing unemployment insurance (UI). This asymmetric information may prevail due to unobservable characteristics or because it is too costly to elicit information. The literature on optimal unemployment insurance generally neglects this unobserved heterogeneity [Karni (1999) provides a comprehensive overview]. ${ }^{1}$ It mainly focuses on the trade-off between setting incentives to search and insurance aspects of providing benefits to a representative agent. ${ }^{2}$ This is remarkable since self-selection from a menu of contracts is common practice when dealing with heterogeneous populations in the design of health insurance or car insurance markets.

In this paper, we ask whether offering a menu of unemployment insurance contracts is welfare improving and what the optimal contracts look like. We adopt a multi-period contract theoretical framework, introduced by Shavell and Weiss (1979) and later refined and simulated by Hopenhayn and Nicolini (1997). We extend their representative agent framework to encompass heterogeneity in the job search technology, i.e., we introduce types of agents that differ with respect to their probability of finding a job when controlling for search effort. We compute the optimal menu of UI contracts offered by a UI agency from which each agent selects his preferred contract.

As HN, our paper technically builds on recursive solutions of repeated games and principal-agent problems as analyzed in the papers by Spear and Srivastava (1987), Thomas and Worrall (1990),

\footnotetext{
${ }^{1}$ Exceptions are Mortensen (1983) and Wang and Williamson (2002). For a discussion see below.

${ }^{2}$ Atkinson and Micklewright (1991) and Meyer (1995) survey the empirical literature that documents disincentive effects from providing UI.
} 
Abreu, Pearce, and Stacchetti (1990) [APS], Atkeson and Lucas (1992) and Chang (1998). However, our combination of adverse selection with repeated moral hazard has not been studied yet. Furthermore, concerning the adverse selection problem, agents' types, which parameterize their chances of finding a job, are drawn only once but affect their chances in all future periods. This assumes that differences in the ability of finding a job are permanent. Although we assume full commitment, there is no "natural" way to state such a problem recursively. The permanent heterogeneity in our model implies that the first period is distinct from the following periods. Both the adverse selection incentive constraints and the entitlement constraints in the principal's problem have to hold only in the first period whereas moral hazard incentive constraints hold in every period. As in Fernandes and Phelan (2000) additional state variables that restrict the planner's choices have to be added.This choice has to ensure that the problem remains computationally tractable. We include the remaining expected life-time utility guaranteed by a contract to an agent as state variables, one for each type. To keep track of the incentive constraints we add, for each type, the remaining expected life-time utility if he had chosen a different contract. We then proceed in two steps. First we give separate recursive formulations of cost minimization problems for each type. This generates optimal contracts implementing previously specified entitlements. Second we state the overall problem by merging these separate cost minimization problems.

In the numerical solution procedure we face a well-known difficulty in the recursive contracts literature building on the APS methodology, namely the efficient calculation of the sets of "sustainable outcomes". 3 Our main theorem provides a precise analytical characterization of the set of sustainable outcomes in our setup. This characterization renders a numerical implementation feasible. In particular, we prove certain topological properties of the set of sustainable outcomes that ensure an efficient computation that considerably improves in terms of accuracy upon previous algorithms [compare e.g. section 8 of Chang (1998)].

\footnotetext{
${ }^{3}$ This terminology was introduced by APS. It describes the sets of contracts that are implementable taking into account future choices which again have to take into account initial choices and so on and so forth.
} 
Our main theorem allows us to compute the optimal menu of UI contracts numerically and thus to answer the following questions. First, under which circumstances will it be optimal to offer only one UI contract to all agents - a situation that resembles real world UI schemes in most countries? We can show (under fairly general conditions) that in the optimum separating contracts should be offered. Second, are all optimal UI contracts decreasing, as in the representative-agent setup [for an overview, see Fredriksson and Holmlund (2003)]? We investigate the shape of optimal contracts in a framework where two types of agents (good and bad) face different hazard rates of finding a job (given a certain search effort). We show that the contract for the good searcher has a decreasing benefit profile, as the one he would be offered in a pure moral hazard environment. In contrast, the contract of the bad searcher will tend to have an upward-sloping benefit profile. Finally, we provide a comparative-statics analysis of changes in various parameters and give a detailed intuition of our results.

In the optimal-UI literature the issue of adverse selection has been raised first by Mortensen (1983) who applies the seminal Rothschild and Stiglitz (1976) paper to UI. His analysis is, however, static and does not include search incentives. Wang and Williamson (2002) present a numerical welfare analysis of UI in a dynamic economy with moral hazard and heterogeneous agents. Their focus is the effect of (full and partial) experience rating on optimal UI. In contrast in our model agents choose from a set of different UI contracts offered by the principal. Hopenhayn and Nicolini (2001) address the issue of heterogeneity of agents in a two period model of UI similar to their earlier work. They assume, however, that the agents' type is observable and contractible.

The paper is organized as follows. In section 2 the model is introduced and our assumptions are motivated. In section 3 the analytical results are presented. In section 4 the model is solved numerically; we also present an extensive comparative statics exercise. Section 5 discusses various possible extensions of our model. Proofs are given in the appendix. 


\section{Model}

Our framework for analyzing UI is a dynamic principal-agent model. The principal represents the government (or UI agency) providing social insurance.

The agents' problem The agents are unemployed workers searching for a job. There is a continuum of agents, modelled by the unit interval. The agents are of two types, differing in their opportunities of finding a new job. The differences between the two types of agents in their search technology is expressed by agent $i$ 's probability of remaining unemployed $p_{i}(a)$, that is a function of the search effort $a$ he exerts (we will sharpen the notion of heterogeneity formally below). They have private information about their types. The fraction of agents of type B ("bad searcher") $q$ and of type G ("good searcher") $1-q$ are common knowledge. Except for the difference in search technologies (as formalized below) we assume that agents are identical. In particular, we assume that both agents are equally risk-averse, they enjoy the same utility $u($.$) from consumption. Also, we assume that once an agent$ has been employed, he will keep his job with a fixed wage rate $w$ until his death in period $T .{ }^{4}$ We can thus calculate an employed agent's total expected lifetime utility in period $t$ as:

$$
W_{t}=\sum_{l=t}^{T} \beta^{l-t} u(w),
$$

where $\beta$ is the common discount factor.

Unemployed agents receive possibly time-varying UI benefits. We denote by $b_{t}$ the benefit received in period $t$. Given any benefit scheme $\left\{b_{1}, \ldots, b_{T}\right\}$, the agent chooses his search effort $a_{t}$ in any period in which he is unemployed. In his decision to increase his search effort he faces a trade-off between increasing search costs on

\footnotetext{
${ }^{4}$ Technically, the assumption that both agents get the same wage is in no way important for our analysis, it is introduced for the sake of simplicity. In the solution to our model we could keep track of the impact of different wages for $B$ and $G$ on the search incentives; qualitatively, the results would not change.
} 
the one hand and an increasing reemployment probability on the other. The total lifetime utility he expects when remaining unemployed is the key variable determining this decision. We denote by $V_{t+1}^{i}$ an unemployed agent's total expected lifetime utility in period $t+1$, where $i=B, G$. Moreover, in the sequel we will denote by $z_{t}=u\left(b_{t}\right)$ the utility value of consuming benefit $b_{t}$. Thus we can state agent $i$ 's problem $(i=B, G)$ in period $t$ recursively by:

$$
V_{t}^{i}=\max _{a} z_{t}-a+\beta\left[p_{i}(a) V_{t+1}^{i}+\left(1-p_{i}(a)\right) W_{t+1}\right],
$$

where we assumed that effort enters utility linearly. Recall that $W_{t+1}$ is an employed agent's expected lifetime utility where employment starts in $t+1$. We denote by $\hat{a}_{t}^{i}$ the decision of agent $i$ :

$$
\hat{a}_{t}^{i}=\operatorname{argmax}_{a} z_{t}-a+\beta\left[p_{i}(a) V_{t+1}^{i}+\left(1-p_{i}(a)\right) W_{t+1}\right] .
$$

The principal will have to take into account the decisions by the agents when designing contracts for them. These constraints are moral hazard incentive constraints, (MH-IC).

The principal's problem The principal's objective is to minimize the cost of providing a certain "level" of insurance by the design of (a menu of) optimal contracts for the agents. Hereby the current cost function $c($.$) is the inverse of the agent's utility$ function: $c\left(z_{i}\right) \equiv u^{-1}\left(z_{i}\right)=b_{i}$. We will call $\left\{z_{1}^{i}, \ldots, z_{T}^{i}\right\}$ a contract designed for agent $i$ (with $i=B, G$ ). We will often use the terms contract $b$ and contract $g$ to denote the contracts designed for agents $B$ and $G$ respectively. Agents choose one contract from the offered contracts in period 0 . We assume that the principal can fully commit to the contract promised in period zero. The following property of $c($.$) is implied by monotonicity and strict$ concavity of an agent's utility function $u($.$) , i.e. risk aversion.$

Condition 2.1 The cost function c(.) is increasing and strictly convex.

In his minimization problem, the principal has to take into consideration the different reemployment probabilities of the agents. Furthermore, he has to take into account the following constraints: 
First, as mentioned above, he has to take into consideration the agents' decision problem, i.e. the moral hazard incentive constraints. Second, the principal has to guarantee incentive compatibility due to adverse selection, i.e., he has to ensure that each agent chooses the contract designed for him. Third, he has to respect the entitlement $\underline{V}$ (i.e. total expected lifetime utility) the contracts should at least guarantee to agent B and G respectively. This entitlement can be interpreted as the "level of insurance" the principal is willing to guarantee. Any value of the entitlement $\underline{V}$ can be mapped one-to-one to a "certainty equivalent replacement rate", i.e., to a percentage of the wage $w$ which is consumed every period and which provides lifetime utility of exactly $\underline{V}$. This interpretation will be used to calibrate reasonable values of $\underline{V}$ later. The principal's problem can thus be stated as:

$$
\begin{array}{r}
\min _{\left\{z_{1}^{B}, \ldots, z_{T}^{B}\right\},\left\{z_{1}^{G}, \ldots, z_{T}^{G}\right\}} q\left[c\left(z_{1}^{B}\right)+\beta p_{B}\left(\hat{a}_{1}^{B}\right)\left[c\left(z_{2}^{B}\right)+\beta p_{B}\left(\hat{a}_{2}^{B}\right)\left[c\left(z_{3}^{B}\right)+\ldots\right] \ldots\right]\right]+ \\
(1-q)\left[c\left(z_{1}^{G}\right)+\beta p_{G}\left(\hat{a}_{1}^{G}\right)\left[c\left(z_{2}^{G}\right)+\beta p_{G}\left(\hat{a}_{2}^{G}\right)\left[c\left(z_{3}^{G}\right)+\ldots\right] \ldots\right]\right]
\end{array}
$$

subject to the entitlement constraints (EC)

$$
\begin{aligned}
& V_{1}^{b, B} \geq \underline{V}, \\
& V_{1}^{g, G} \geq \underline{V},
\end{aligned}
$$

and the adverse selection incentive constraints (AS-IC)

$$
\begin{aligned}
& V_{1}^{b, B} \geq V_{1}^{g, B}, \\
& V_{1}^{g, G} \geq V_{1}^{b, G} .
\end{aligned}
$$

The hat on the $a$ 's describing the choices of effort of the agents, $\hat{a}$, indicate that the principal respects the moral hazard incentive constraints (MH-IC).

In the formulation of the principal's problem, $V_{t}^{j, i}$ denotes total expected lifetime utility in period $t$ for the unemployed agent $i$ ( $i=$ $B, G)$ if he selects contract $j(j=b, g)$. The superscript $j$ indicates for which agent the contract is designed, i.e., contract $b$ is designed for agent $B$ and contract $g$ for agent $G$. A priori both agents 
can of course choose either contract before period 1 . The ASIC constraints ensure that they will in fact choose the contract designed for them. The EC constraints guarantee that the chosen contract gives the promised utility. Both the $V_{t}^{j, i}$ s and the $\hat{a}_{t}^{i} \mathrm{~s}$ can be calculated recursively from the array of equations 1 and 2 given a contract.

Remark 2.2 If agents are homogeneous in their search costs, i.e. they all have the same $p_{i}(a)$, then the setup is identical to the one considered by Shavell and Weiss (1979) (except for the finite time dimension).

To make our problem interesting, the initial entitlements to total expected lifetime utility have to be below the total lifetime utility from work. If the entitlements $V_{t}^{i, j}$ are higher than $W_{t}$ for any period posterior to one in that the unemployed agents exert search effort, the efforts would necessarily be zero, and thus the probability of remaining unemployed $p_{i}$ would be 1 .

Condition 2.3 The utility entitlement of the unemployed agent is below the one guaranteed by lifetime work: $\underline{V}<W_{1}$.

We will see in the sequel that this condition guarantees that all optimal $V_{t}^{i, j}$ are smaller than $W_{t}$.

Formalization of the Agents' Heterogeneity Now we formalize in detail the idea that agents differ in their reemployment probabilities. To develop the model model formally we first make some standard technical assumptions on the $p_{i}($.$) functions.$

Condition 2.4 The probability of remaining unemployed $p_{i}(a)$ of agent $i$ :

1. Smoothness: $p_{i}(a) \in C^{\infty}(\mathbf{R})$.

2. Monotonicity and strict Convexity: $p_{i}^{\prime}(a)<0, p_{i}^{\prime \prime}(a)>0$.

3. Boundary conditions: $p_{i}(0)=1, \lim _{a \rightarrow \infty} p_{i}(a)=0$.

4. Inada conditions: $\lim _{a \rightarrow 0} p_{i}^{\prime}(a)=-\infty, \lim _{a \rightarrow \infty} p_{i}^{\prime}(a)=0$. 
Condition 2.4 ensures that the agents' problem (1) always has a unique interior solution that can be characterized by a first order condition.

The basic difference between the two types of agents in their search technology is expressed by agent $i$ 's probability of remaining unemployed $p_{i}(a)$, that is a function of the search effort $a$ he exerts:

Condition 2.5 Given the same effort, type B has a higher probability of remaining unemployed than type $G$ : $p_{B}(a)>p_{G}(a)$.

\section{Condition 2.6 (Spence-Mirrlees property)}

$$
\frac{\partial V_{t}^{B}}{\partial z_{t+s}}-\frac{\partial V_{t}^{G}}{\partial z_{t+s}}>0
$$

for all $t$ with $1 \leq t \leq T$ and $s$ with $1 \leq s \leq T-t$.

Condition 2.6, which is standard in classical contract theory, sharpens the basic concept of a bad or good searcher. It implies that faced with the same contract - the bad searcher may exert a higher search effort than the good searcher under this contract, but his effort will not be so high that his chance of finding a job exceeds the good searcher's chance:

Lemma 2.7 Condition 2.6 holds if and only if agents facing the same contract $\left\{z_{1}, \ldots, z_{T}\right\}$, in equilibrium exert efforts such that $p_{B}\left(a_{t}^{B}\right)>p_{G}\left(a_{t}^{G}\right)$.

Condition 2.4 to 2.6 will be used in all results that follow. For our first result - on the optimality of separating the types - we need one more assumption on $p_{i}($.$) . As we mentioned above, con-$ dition 2.4 ensures that the agent's choice of effort from (1) can be characterized by the following first order condition:

$$
p_{i}^{\prime}\left(a_{t}^{i}\right)=\frac{1}{\beta\left(V_{t+1}^{i}-W_{t+1}\right)} .
$$

Equation (8) establishes a one-to-one and smooth relation between $V_{t}^{i}$ and $a_{t}^{i}$. We can therefore define the following function for the 
next-to-last period:

$$
\pi_{i}\left(z_{T}\right) \equiv p_{i}\left(a_{T-1}^{i}\right)=p_{i}\left(\left(p_{i}^{\prime}\right)^{-1}\left(\frac{1}{\beta\left(z_{T}-u(w)\right)}\right)\right)
$$

So $\pi_{i}\left(z_{T}\right)$ is agent $i$ 's probability of remaining unemployed when facing benefit utility $z_{T}$ in the last period. It is clear that $\pi_{i}$ is increasing. We formulate a condition on the elasticity of $\pi_{i}$ with respect to $z_{T}$, which - of course - implicitly puts restrictions on the choice of $p_{i}($.$) .$

Condition 2.8 1. The marginal probability of remaining unemployed $\frac{\partial \pi_{i}(z)}{\partial z}$ of agent $i$ facing promised utility $z$ is greater for agent $G$ than for agent $B$ :

$$
\frac{\partial \pi_{G}(z)}{\partial z}>\frac{\partial \pi_{B}(z)}{\partial z}
$$

2. If the marginal probabilities $\frac{\partial \pi_{i}(z)}{\partial z}$ of agent $G$ and $B$ are equal for two utility values $z^{G}$ and $z^{B}$, then the utility of $G, z^{G}$, must be smaller than the utility of $B, z^{B}$ :

$$
\frac{\partial \pi_{G}\left(z^{G}\right)}{\partial z}=\frac{\partial \pi_{B}\left(z^{B}\right)}{\partial z} \Rightarrow z^{G}<z^{B} .
$$

What is the economic content of Condition 2.8? The first part says that agent $\mathrm{G}$ reacts more strongly to a change in the promise $z$ than agent B. In other words: The probability of finding a new job depends more critically on the UI benefit promise in the case of agent $\mathrm{G}$ than in the case of agent B. Note that here we compare agents $G$ and $B$ that face the same contract. The second part of condition 2.8 says, that whenever the reaction is equal, then agent G must face a lower promise than agent B. Summarizing condition 2.8 we can say that the "incentive sensitivity" of agent $G$ is higher than the one of agent B.

What role do conditions 2.6 to 2.8 play in our analysis? The model presented in this paper incorporates two different paradigms, hidden information and (repeated) hidden action. Condition 2.6 is the typical technical assumption in hidden information models. Condition 2.8 is a condition that ensures in our setup a feature of 
(pure) repeated hidden action that has been analyzed numerically in Pavoni (2000) and discussed in Hopenhayn and Nicolini (2001): In the full information case the decline of the UI benefits over time is sharper for better searchers. Loosely speaking, conditions 2.6 and 2.8 ensure that our model exhibits the "standard" behavior of a pure hidden information model and a pure hidden action model. We will see in section 4 that both conditions will be met in our functional specification of our simulation.

\section{Theoretical Results}

In this section we develop a characterization of the solution to the principal's problem. First, we will however turn to a standard question in contract theory.

Pooling is Not Optimal The first question we ask is whether and under what circumstances it is actually optimal to offer two contracts in order to screen the agents. The answer gives a first indication that it may indeed be relevant to consider the cost saving potential of a differentiated UI.

Proposition 3.1 There exists a solution to the Principal's Problem. If Condition 2.8 holds, any solution is separating.

What makes the theorem very appealing from a more applied perspective is the fact that our numerical implementation with CRRA utility shows that the good searcher typically reacts more sensitively (in the sense of condition 2.8) to the search incentives than the bad searcher. Considering this numerical result as robust we could claim that the UI agency has a definite potential for cost-saving by switching from offering only one to offering two UI contracts.

But what should the optimal contracts look like? The answer to this question is not evident: We can neither apply Shavell and Weiss (1979) solution directly, since we should expect the influence of hidden information on the optimal design of the contracts, nor can we apply standard solutions of adverse selection models that do not incorporate repeated hidden action. Moreover we cannot 
hope to find a direct recursive formulation of the problem, because both the adverse selection incentive constraints and the entitlement constraints in the principal's problem have to hold only in the first period.

The Solution of the Model In the sequel, we develop a characterization of the solution to the principal's problem in Propositions 3.2, 3.4 and 3.8. The strategy is as follows: We first look at each contract separately ignoring for a moment the issue of selfselection. In Proposition 3.2 we give a recursive formulation of the problem of finding a cost-minimizing contract that provides agent $B$ and agent $G$ with two arbitrarily specified levels of ex-ante lifetime utility (a pair of first-period entitlements) if both of them choose this contract. The goal of this proposition is to summarize the cost minimization problem in a compact way, i.e., to give two separate recursive formulations, one for each contract. In this formulation the pairs of entitlements (one pair for each contract) and their evolution over time serve as state variables of the problem (compare $\mathrm{HN}$ ).

The question left open by proposition 3.2 is which pairs of entitlements are actually jointly feasible under a given contract (still ignoring the issue of self-selection). The answer to this question has to take into account the choices of effort by the agents induced by the contract under consideration as well as the laws of motion for the entitlements. Proposition 3.4 gives a precise theoretical and numerically useful description of the correspondence $\Gamma_{t}$ mapping pairs of entitlements of the agents today $\left(V_{t}^{B}, V_{t}^{G}\right)$ to jointly feasible policy options $\left(z_{t}, V_{t+1}^{B}, V_{t+1}^{G}\right)$, i.e., the benefit today and the entitlements for tomorrow. This proposition is the main theoretical result of the paper and serves as the cornerstone of our recursive numerical implementation. We can now calculate the cost of a given contract that provides agents with any feasible pairs of first period entitlements.

Finally, we return to address the issue of self-selection. We merge the two separately solved cost minimization problems and state the original adverse selection problem faced by the principal as a four-dimensional minimization problem in the (two pairs of) entitlements of the first period. In Proposition 3.8 we further 
simplify the minimization problem by showing that the entitlement constraint of $G$ must be slack, and that the entitlement constraint of $B$ and the adverse selection incentive constraint of $G$ must be binding at the solution. This reduces the dimension of the problem from four to two which allows us to solve the problem with high numerical accuracy.

We begin the characterization of the solution by the recursive formulation of contract b, i.e. the contract designed for agent B. Of course contract g can be described similarly. As usual in adverse selection problems, we anticipate that only agent $\mathrm{B}$ will choose contract $\mathrm{b}$ in the end and thus stochastically discount costs at his rate. For the time being, the lifetime (or first period) entitlements of contract b for agent B and agent $\mathrm{G}, V^{b, B}$ and $V^{b, G}$, are taken as given. Their optimal values for given entitlement constraints (EC) from the original problem will be calculated in Proposition 3.8 below. The recursive formulation takes the form of a (finitedimensional) Bellman Equation: The principal minimizes the costs of paying out a benefit worth $z_{t}$ (in utility units) today and promising entitlements $V_{t+1}^{b, B}$ and $V_{t+1}^{b, G}$ for tomorrow. In doing so, he has to observe the entitlements of B and G today, $V_{t}^{b, B}$ and $V_{t}^{b, G}$, that serve as state variables of the problem. A law of motion connects the state and the choice variables. We denote the set of possible choices $\left(z_{t}, V_{t+1}^{b, B}, V_{t+1}^{b, G}\right)$ in state $\left(V_{t}^{b, B}, V_{t}^{b, G}\right)$ by $\Gamma_{t}\left(V_{t}^{b, B}, V_{t}^{b, G}\right)$. This correspondence will be characterized later in Proposition 3.4. Moreover, the choices of effort $a_{t}^{i}$ from the agents' problem facing the promised entitlement $V_{t+1}^{i}$ for tomorrow, i.e. the MH-IC, are taken as given by the principal. The recursive formulation is completed by two boundary conditions: The first period entitlements $V_{1}^{b, B}$ and $V_{1}^{b, G}$ of course have to equal the promised ex ante lifetime utilities $V^{b, B}$ and $V^{b, G}$ respectively. In the last period, the entitlements $V_{T}^{b, B}$ and $V_{T}^{b, G}$ have to take the value of the last period benefit $z_{T}$. To see this, recall that we consider agents who chose the same contract, namely contract b. So both get a benefit of $z_{T}$ in the last period. But in the last period, unlike all other periods, there is no way of splitting the promise for that period into a benefit in that period and a promise one period later because there is no period later. 
Thus we can state the following proposition without proof: ${ }^{5}$

Proposition 3.2 (Recursive Formulation of Contract b) The cost functions for contract $b$, guaranteeing an ex ante lifetime utility of $V^{b, B}$ to agent $B$ and $V^{b, G}$ to agent $G$, has the following recursive form:

$C_{t}^{B}\left(V_{t}^{B}, V_{t}^{G}\right)=\min _{\left\{z_{t}, V_{t+1}^{B}, V_{t+1}^{G}\right\} \in \Gamma_{t}\left(V_{t}^{B}, V_{t}^{G}\right)} c\left(z_{t}\right)+\beta p_{B}\left(a^{B}\right) C_{t+1}^{B}\left(V_{t+1}^{B}, V_{t+1}^{G}\right)$

subject to

Law of motion for contract b (LOM)

$$
\begin{aligned}
& z_{t}-a^{B}+\beta\left[p_{B}\left(a^{B}\right) V_{t+1}^{B}+\left(1-p_{B}\left(a^{B}\right)\right) W_{t+1}\right]=V_{t}^{B} \\
& z_{t}-a^{G}+\beta\left[p_{G}\left(a^{G}\right) V_{t+1}^{G}+\left(1-p_{G}\left(a^{G}\right)\right) W_{t+1}\right]=V_{t}^{G},
\end{aligned}
$$

Choice of effort in contract b (MH-IC)

$$
\begin{aligned}
& a^{B}=\operatorname{argmax}_{a} z_{t}-a+\beta\left[p_{B}(a) V_{t+1}^{B}+\left(1-p_{B}(a)\right) W_{t+1}\right] \\
& a^{G}=\operatorname{argmax}_{a} z_{t}-a+\beta\left[p_{G}(a) V_{t+1}^{G}+\left(1-p_{G}(a)\right) W_{t+1}\right]
\end{aligned}
$$

as well as the boundary conditions

$$
\begin{aligned}
V_{1}^{B} & =V^{b, B} \\
V_{1}^{G} & =V^{b, G} \\
V_{T}^{B} & =z_{T} \\
V_{T}^{G} & =z_{T} .
\end{aligned}
$$

The correspondence $\Gamma_{t}$ maps into the values of jointly feasible $z_{t}$, $V_{t+1}^{B}, V_{t+1}^{G}$ given the pair of entitlements $\left(V_{t}^{B}, V_{t}^{G}\right)$.

Remark 3.3 Of course the contract $g$ for agent $G$ takes the same form as contract $b$ for agent $B$ in Proposition 3.2, whereby necessary adaptations in the entitlements etc. are obvious.

\footnotetext{
${ }^{5}$ A proof by induction over $T$ is straightforward. The recursive formulation can thus be understood as a way of summarizing terms in the principal's problem above.
} 
In order to make use of Proposition 3.2, we actually have to be able to calculate the correspondence $\Gamma_{t}(.,$.$) as precisely as possi-$ ble. This is particularly important for any numerical application of the recursive formulation. The following proposition gives a characterization of theoretical properties of $\Gamma_{t}$ that are indispensable for a satisfactory approach to calculate $\Gamma_{t}$ numerically. For technical reasons we distinguish between the case where the utility $z_{t}$ from consuming the UI benefit is bounded from below from the case where it is not. We will discuss this and other issues after stating the proposition. ${ }^{6}$

Proposition 3.4 (Characterization of $\Gamma_{t}$ ) The following formulas characterize the correspondence $\Gamma_{t}\left(V_{t}^{B}, V_{t}^{G}\right)$, that gives all feasible policy options for given entitlements $V_{t}^{B}$ and $V_{t}^{G}$ in period $t$. Note that we allow for a lower bound $\underline{z}$ on the utility value of the benefit $z_{t}$. The reason will be explained in Remark 3.5.

1. Be $t \leq T$ and let $z_{t} \geq \underline{z}$ (where $\underline{z}$ may take the value $-\infty$ ). Then there exists a lower bound $\underline{V}^{G}$ and so that for $\underline{V}^{G} \leq$ $V_{t}^{G} \leq W_{t}$ :

$$
\Gamma_{t}\left(V_{t}^{B}, V_{t}^{G}\right)= \begin{cases}\left\{z_{t}(a), V_{t+1}^{B}(a), V_{t+1}^{G}(a)\right\}_{a \in[\underline{a}, \bar{a}]\left(V_{t}^{B}, V_{t}^{G}\right)} & : \quad V_{t}^{B} \in\left[\underline{V}_{t}^{B}\left(V_{t}^{G}\right), \bar{V}_{t}^{B}\left(V_{t}^{G}\right)\right] \\ \emptyset & : \text { else }\end{cases}
$$

The jointly feasible values $z_{t}(a), V_{t+1}^{B}(a)$ and $V_{t+1}^{G}(a)$ are differentiable functions.

The boundary functions $\underline{a}\left(V_{t}^{B}, V_{t}^{G}\right), \bar{a}\left(V_{t}^{B}, V_{t}^{G}\right)$ and $\underline{V}_{t}^{B}\left(V_{t}^{G}\right)$, $\bar{V}_{t}^{B}\left(V_{t}^{G}\right)$ are continuous. They are depend on the value of $\underline{z}$. For $V_{t}^{G}$ below $\underline{V}^{G}$, the correspondence $\Gamma_{t}\left(V_{t}^{B}, V_{t}^{G}\right)$ is the empty set.

2. In period $T-1$ the correspondence $\Gamma_{T-1}\left(V_{T-1}^{B}, V_{T-1}^{G}\right)$ takes the same form as in 1 with only one possible parameter value a, i.e. only one choice $\left\{z_{T-1}(a), V_{T}^{B}(a), V_{T}^{G}(a)\right\}$.

\footnotetext{
${ }^{6}$ The notation $a \in[\underline{a}, \bar{a}]\left(V_{t}^{B}, V_{t}^{G}\right)$ means that the boundaries $\underline{a}$ and $\bar{a}$ are functions $\underline{a}\left(V_{t}^{B}, V_{t}^{G}\right)$ and $\bar{a}\left(V_{t}^{B}, V_{t}^{G}\right)$.
} 
We have two technical remarks on Proposition 3.4.

Remark 3.5 The upper bound $W_{t}$ on $V_{t}^{B}$ is artificial: Of course the principal can ensure lifetime utilities above the value of secure lifetime income from work. However, this cannot be optimal, since it reduces the search effort to zero, and in view of Condition 2.3 we exclude lifetime utilities above $W_{t}$ from our considerations.

Remark 3.6 The lower bound on $z_{t}$ in Proposition 3.4 is introduced for technical reasons: Some utility functions map onto the real line $\mathbf{R}$, some only onto the half-line $\mathbf{R}_{+}$. An example of the former kind are CARA utility functions, one of the latter CRRA utility functions with risk aversion smaller than one.

With Proposition 3.4 at hand, we can define precisely the notion of feasibility in our model: A pair of entitlements $\left(V_{t}^{B}, V_{t}^{G}\right)$ is called jointly feasible if the set $\Gamma_{t}\left(V_{t}^{B}, V_{t}^{G}\right)$ is non-empty.

Let us now be more specific why this proposition is so important for our purposes. Models including repeated moral hazard, as ours, have been discussed in the framework of a strand of literature building on Spear and Srivastava (1987), Thomas and Worrall (1990), Abreu, Pearce, and Stacchetti (1990) [APS], Atkeson and Lucas (1992) and Chang (1998). We are not bound to provide a detailed discussion of this literature, the following remark, however, relates proposition 3.4 to it.

Remark 3.7 The sets of jointly feasible entitlements are the finitedimensional analogue of the set of sequential equilibrium payoffs (of the agents' game) in the infinite-dimensional framework of $A P S$ or the set of sustainable outcomes in the (again infinitedimensional) framework of Chang (1998).

By introducing entitlements, marginal utilities or sequential equilibrium outcomes as state variables - instead of "intuitive" state variables - we inevitably run into the difficulty of defining precisely the sets of possible values these state variables can take. APS and Chang (1998) characterize these sets as the largest fixed point of a set operator. Moreover, they show that the fixed point 
can be obtained by a fixed-point iteration of sets. This is theoretically sound. However, it does not provide an entirely satisfactory description of the sets nor the definite algorithm to calculate them numerically, in particular if the state space is more than onedimensional. In fact, the numerical determination of these sets may be a tricky issue in simulations of models building on these methods. In Proposition 3.4 we give - for our model - a satisfactory description of the sets of the state variables: The boundaries of the sets are continuous functions. In particular, the sets are compact, connected and contractible. In the next section we will point out that this is crucial for the numerical implementation of our solution. Moreover, Proposition 3.4 states that the principal's choice problem in a given period is essentially one-dimensional (in the sense that the correspondence describes a smooth one-dimensional path in the three-dimensional real space with this path being parameterized in $a)^{7}$

After this methodological digression, we return to the solution of the principal's problem. Given proposition 3.2 it can be stated as follows:

$$
\begin{aligned}
& \left.\min _{V^{b, B}, V^{b, G}, V^{g, B}, V^{g, G}} q C_{1}^{B}\left(V^{b, B}, V^{b, G}\right)+(1-q) C_{1}^{G}\left(V^{g, B}, V^{q,(15)}\right)\right) \\
& \text { s.t. } \quad \Gamma_{1}\left(V^{b, B}, V^{b, G}\right) \neq \emptyset \\
& \Gamma_{1}\left(V^{g, B}, V^{g, G}\right) \neq \emptyset \\
& V^{b, B} \geq V^{g, B} \\
& V^{g, G} \geq V^{b, G} \\
& V^{b, B} \geq \underline{V} \\
& V^{g, G} \geq \underline{V} \text {. }
\end{aligned}
$$

This is a four dimensional minimization problem, and thus still rather complex. However, as in the case of standard adverse selection problems (compare e.g. the book by Laffont and Martimort (2002), Chap. 2), we are able show that agent B's entitlement constraint and agent G's adverse selection incentive constraint must be binding, and that agent G's entitlement constraint must be slack at the solution. And so we finally characterize the solution

\footnotetext{
${ }^{7}$ Except for the next-to-last period, where there is only one choice left.
} 
to the principal's problem as follows:

Proposition 3.8 (Solution of the Principal's Problem) For values of $\underline{V}$ that are not too low, solutions to the principal's problem are solutions to the simplified problem:

$$
\begin{array}{ll} 
& \min _{V^{g, B}, V^{g, G}} q C_{1}^{B}\left(\underline{V}, V^{g, G}\right)+(1-q) C_{1}^{G}\left(V^{g, B}, V^{g, G}\right) \\
\text { s.t. } & \underline{V} \geq V^{g, B}
\end{array}
$$

The qualification "not too low" is needed to avoid corner solutions. In the simulation we found that all values of $\underline{V}$ corresponding to reasonable levels of insurance were high enough (compare the proof in the appendix and footnote 9 in Section 4.1).

Two Corollaries Two corollaries ensue from proposition 3.8. The set-up considered by Shavell and Weiss (1979) will be our benchmark, i.e. a set-up where the principal knows the type of the agent and sets the benefits to give optimal search incentives. We will call it the pure moral hazard environment. About the contract for type B we learn:

Corollary 3.9 Type $B$ receives the minimal entitlement utility $\underline{V}$. His contract is distorted with respect to the optimal contract in a pure moral hazard environment such that its value $V^{b, G}$ for type $G$ is reduced.

In the case of the contract for type $\mathrm{G}$, we deduce:

Corollary 3.10 1. Type $G$ receives an information rent, i.e. the utility $V^{g, G}$ that his contract provides him with is greater than $\underline{V}$.

2. If the adverse selection incentive constraint of the bad searcher (18) is slack at the solution, his contract is identical to the optimal contract in the pure moral hazard environment (given the level of entitlement $V^{g, G}$ ). 
In our numerical simulations, we found that the adverse selection incentive constraint of the bad searcher 18 was always slack.

We thus recover the rent extraction/efficiency trade-off from a simple adverse selection model without moral hazard (cf. chapter 2 of Laffont and Martimort (2002)), where efficiency here is the search efficiency of agent B.

The corollaries provide first economic insights as to how the unemployment agency should design optimal UI contracts. Further economic aspects will be discussed after a simulation of optimal contracts in the next section.

\section{Simulation}

\subsection{Computational Strategy}

The simulation closely follows Proposition 3.2, Proposition 3.4 and Proposition 3.8 in Section 2. The first part of the simulation calculates the correspondence $\Gamma_{t}$ by backward induction. As in Proposition 3.4, we use the entitlement of agent $G V_{t}^{G}$ as a parameter for the upper and lower bound on the entitlement $V_{t}^{B}$ for agent B. We introduce a grid on $V_{t}^{G}$ and then calculate the bounds on $V_{t}^{B}$ by a bracketing procedure.

More precisely, for a given tuple $\left(V_{t}^{B}, V_{t}^{G}\right)$ of state variables, we check whether the corresponding path of choice variables $\left(z_{t}(a), V_{t+1}^{B}(a), V_{t+1}^{G}(a)\right)$ (compare the proof of Proposition 3.4, Appendix C) intersects the set of jointly feasible values $\left(z_{t}, V_{t+1}^{B}, V_{t+1}^{G}\right)$ as determined in the previous induction step. As defined in the previous section, by "jointly feasible" we refer to tuples $\left(V_{t+1}^{B}, V_{t+1}^{G}\right)$ such that $\Gamma_{t+1}\left(V_{t+1}^{B}, V_{t+1}^{G}\right)$ is non-empty; in the case of $z_{t}$, we only have to check whether it is above the lower bound $z$. By proposition 3.4, we know that for each $V_{t}^{G}$ (within the limits of feasible entitlements for $\mathrm{G}$ ) there exits a $\underline{V}_{t}^{B}\left(V_{t}^{G}\right)$ and $\bar{V}_{t}^{B}\left(V_{t}^{G}\right)$ that limits the range of $V_{t}^{B}$ jointly feasible with $V_{t}^{G}$. Since for every $V_{t}^{G}$ the set of jointly feasible $V_{t}^{B}$ is one interval, we can "encircle" $\underline{V}_{t}^{B}$ (and, separately, $\bar{V}_{t}^{B}$ ) by values of $V_{t}^{B}$ above and below and then calculate the bound by a (highly precise) bracketing procedure. It is thus proposition 3.4 that guarantees that our algorithm calculates a characterization 
of the set of jointly feasible entitlements $\left(V_{t}^{B}, V_{t}^{G}\right)$ by stating that the set of jointly feasible entitlements $\left(V_{t+1}^{B}, V_{t+1}^{G}\right)$ is compact and connected.

As we have pointed out in the preceding section (compare remark 3.7 and the following discussion), the virtue of proposition 3.4 lies in a more "precise" characterization of the sets of jointly feasible entitlements (the set of "sustainable outcomes" in the terminology of Chang (1998)). It is exactly here in the numerical algorithm where this characterization becomes useful: The description of the set of sustainable outcomes as a fixed-point of a set-operator by APS is mathematically precise, but poses a serious precision problem in numerical applications with more than one state variable (compare the discussion in section 8 of Chang).

The second part of our numerical procedure uses the recursive formulation in Proposition 3.2. It calculates a numerical approximation of the cost functions $C_{t}^{i}\left(V_{t}^{B}, V_{t}^{G}\right)$ based on a solution of the minimization problems in the backward induction of the principal.

More precisely, we cover the domain of $C_{t}^{i}$ (i.e. the set of jointly feasible entitlements $\left(V_{t}^{B}, V_{t}^{G}\right)$ for which $\Gamma_{t}\left(V_{t}^{B}, V_{t}^{G}\right)$ is non-empty) by a large grid. For each tuple $\left(V_{t}^{B}, V_{t}^{G}\right)$ in the grid ("states of the world"), we solve the minimization problem along the path of choice variables $\left(z_{t}(a), V_{t+1}^{B}(a), V_{t+1}^{G}(a)\right)$, i.e. we solve it in $a$. Ignoring the exact value of the limits $\underline{a}$ and $\bar{a}$, we use a bracketing procedure in which we allocate an extremely high cost to $a$ values delivering choice variables outside the set of feasible values. Note that we make use of the characterization of $\Gamma_{t}$ in two ways: First, we rely on the fact that the set of jointly feasible entitlements $\left(V_{t}^{B}, V_{t}^{G}\right)$ is compact and contractible ${ }^{8}$. Second, we exploit the reduction of the number of choice variables from three $\left(z_{t}, V_{t+1}^{B}, V_{t+1}^{G}\right)$ to one $a$.

Finally, each cost function is then approximated as a linear combination of complete Chebychev polynomials by regression (for this standard procedure, compare Judd (1998), Chapters 6.4, 6.12 and 12.8).

\footnotetext{
${ }^{8}$ If it were not contractible there could be holes in the set of jointly feasible state vectors $\left(V_{t}^{B}, V_{t}^{G}\right)$ and we would have to split up the minimization path into several parts - a tedious and hardly tractable task.
} 
In the third part, the approximated cost functions $C_{1}^{i}$ are combined in the objective function of the principal's problem. According to proposition 3.8, we have to solve a two-dimensional minimization problem ${ }^{9}$.

After an initial grid search, the solution is calculated by a Nelder-Mead multidimensional minimization procedure.

\subsection{Calibration of the Model}

In our calibration, we work with a monthly interval. Therefore we set the discount rate to $\beta=0.995$ which corresponds to an annual discount rate of 0.95 . The overall time-spell is a year, i.e. the number of periods is set to $T=12$. As for the probability function, we choose $p_{i}(a)=1-\sqrt{1-\exp \left(-\theta_{i} a\right)}$, where $\theta_{i}$ remains to be determined. We use CRRA utility functions $u(b)=\frac{b^{1-\gamma}}{1-\gamma}$, as common in the UI literature.

Needless to say, the corresponding cost function $c()=.u^{-1}($. meets the convexity condition 2.1:

$$
c(z)=z^{\alpha},
$$

where $\alpha=\frac{1}{1-\gamma} \geq 1$. Also, Conditions 2.4, 2.6 and 2.8 are fulfilled by the probability function $p_{i}(a)^{10}$. So in particular, in our numerical setup the prerequisites of Theorem 3.1 hold.

In the benchmark case, following $\mathrm{HN}$, we set $\alpha=2$ (i.e. risk aversion $\gamma=\frac{1}{2}$ ), which corresponds to intermediate risk aversion on behalf of the agents.

The wage is normalized to be $w=100$, so that unemployment benefits become equal to replacement rates. The lower bound on the UI benefits is set to $\underline{z}=0$, the lowest possible value taken by CRRA utility functions with $0<\gamma<1$. As in the proofs to the propositions, in our simulation we have normalized utility from

\footnotetext{
${ }^{9}$ There is one point to take care of, though: In order to apply proposition 3.8 , we have to ensure that the minimal entitlement $\underline{V}$ is so high that $z_{1}>\underline{z}$ (compare the proof in Appendix C). We ran alternative minimization routines for low values of $\underline{V}$, showing that the assertion holds.

${ }^{10}$ Our probability function $p_{i}(a)$ has a slightly more intricate functional form than the one used by HN $\left(p_{H N}(a)=1-\exp (-r a)\right)$. We have chosen it because the latter does not fulfill the Inada condition (cf. Condition 2.4).
} 
consuming the wage to zero, i.e. all expected lifetime utilities are negative.

We have finally chosen the parameters $\theta_{1}=0.007$ and $\theta_{2}=$ 0.017 to match reasonable escape rates from unemployment in autarky (compare Meyer (1990)): For type B, the bad searcher, this is then $22.7 \%$ per month, for type $\mathrm{G}$, the good searcher, it is $35.8 \%$ per month. As a comparison: HN assume a weekly escape rate in autarky of $10 \%$ as an average for the US, which corresponds to a monthly escape rate of $34.4 \%$. Other choices of parameters will be discussed in the next section, where we give a detailed comparative statics analysis.

Our first figures, figure 2 to 5 , show how the set of feasible entitlements becomes larger and larger along the backward induction. Figures 6 to 14 show optimal UI contracts for different levels of entitlements to the agents and for different values of $q$, the share of the first agents in the population. The entitlement bounds chosen are $\underline{V}=-20,-25,-30$ and -35 (also denoted in the legend). This corresponds to a certainty equivalent of $68.67 \%$, $61.76 \%, 55.21 \%$ and $49.03 \%$ of the wage per period respectively, i.e. the utility of an (unemployed) agent who consumes $X \%$ of the wage for all periods and cannot gain employment. ${ }^{11}$

\subsection{Results}

In this section we will explore the dependence of the optimal contracts on different choices of parameters. Since we know that Theorem 3.1 applies, all solutions are separating. Moreover, for all parameter values we have looked at the incentive constraint of agent B, equation 18 was slack. For contract g designed for the good searcher $\mathrm{G}$ this means according to Corollary 3.10 that it is identical to the one considered by Shavell and Weiss (1979). From their paper (compare also HN and Pavoni (2000)) we know that in our setup the optimal contract for the good type $G$ has a falling benefit profile, induced by moral hazard; and this property

\footnotetext{
${ }^{11}$ In the cases of $\underline{V}=-20,-25$ the UI benefits for agent $\mathrm{G}$ exceed a replacement rate of $100 \%$ in the first periods. This reflects the fact that our model does not incorporate effects of UI on work effort and its impact on employment.
} 
is reflected by our figures. Changes in the parameter values do not qualitatively change contract g; the falling benefit profile is robust, as our theory predicts (for a detailed numerical discussion of the comparative statics of the contract in a pure moral hazard environment see Pavoni (2000)). However, the level of entitlement for $\mathrm{G}$ (and so, in particular, his information rent) varies with the parameters of our model.

We will thus concentrate our discussion on contract $b$ and the information rent for $\mathrm{G}$. The shape of contract $\mathrm{b}$ is determined by two effects:

1. a moral hazard effect ( $\mathrm{MH})$, arising as in the case of type $\mathrm{G}$ from the agents' search problem,

2. an adverse selection effect (AS), arising from the principal's wish to lower the value of the contract for type $\mathrm{G}$ (compare Corollary 3.9) in order to separate the type types.

We know that in the pure $\mathrm{MH}$ environment, benefit schemes are falling. What would agent B's contract look like in a pure $A S$ environment? As a pure adverse selection environment, we consider a set-up where the type of an agent is still hidden information, but the probabilities of remaining unemployed of type $\mathrm{B}$ and $\mathrm{G}$ are fixed constants $p_{B}>p_{G}$. This is then a typical adverse selection problem as discussed in Chapter 2 of Laffont and Martimort (2002). Due to the assumption of full commitment, the dynamics of the contracts is rather simple. Now, as in the case of the full problem (Cf. Corollary 3.10), agent $\mathrm{G}$ receives an information rent, and, given the entitlement $V^{g, G}$, his contract is the first best contract. In the pure AS case, this means his consumption is fully smoothed. Trivially, the following Spence-Mirrlees property holds (as in the full problem):

$$
\frac{\partial V_{t}^{B}}{\partial z_{t+s}}-\frac{\partial V_{t}^{G}}{\partial z_{t+s}}>0 \quad \forall s \geq 1 .
$$

Therefore, in order to separate the two types, the contract for agent $\mathrm{B}$ has to show an increasing benefit scheme. ${ }^{12}$

\footnotetext{
${ }^{12} \mathrm{~A}$ formal derivation of the solution to the principal's problem in a pure adverse selection environment is available upon request.
} 
Information Rents

\begin{tabular}{c|c|rrr}
$\underline{V}$ & Certainty Equivalent & $q=0.2$ & $q=0.5$ & $q=0.8$ \\
\hline \hline-35 & 49.03 & $10.8 \%$ & $18.1 \%$ & $22.2 \%$ \\
-30 & 55.21 & $7.3 \%$ & $13.9 \%$ & $17.0 \%$ \\
-25 & 61.76 & $4.8 \%$ & $10.5 \%$ & $12.9 \%$ \\
-20 & 68.67 & $3.1 \%$ & $7.2 \%$ & $9.9 \%$
\end{tabular}

(rents expressed as percentage increase over minimal entitlement)

Table 1: Information Rents obtained by agent $\mathrm{G}$

Elasticities $\sigma_{p}$ of agent $B$

\begin{tabular}{c|c|rrr}
$\underline{V}$ & Certainty Equivalent & $\theta_{B}=0.004$ & $\theta_{B}=0.007$ & $\theta_{B}=0.010$ \\
\hline \hline-35 & 49.03 & 0.0070 & 0.0116 & 0.0167 \\
-30 & 55.21 & 0.0075 & 0.0131 & 0.0188 \\
-25 & 61.76 & 0.0079 & 0.0139 & 0.0199 \\
-20 & 68.67 & 0.0083 & 0.0146 & 0.0209
\end{tabular}

Table 2: Elasticities of unemployment probability w.r.t. benefit level for agent $B$

So should we expect the benefit scheme for agent B to be rising or falling? Whether the $\mathrm{MH}$ or the AS effect dominates depends on the choice of parameters. In the sequel, we discuss the influence of different parameters on the relative weight of the $\mathrm{MH}$ and the AS effect. As our benchmark, we use the parameterization of the previous subsection $\left[\alpha=2, \theta_{B}=0.007, \theta_{G}=0.017, q=0.5\right]$.

The entitlement bound $\underline{V}$ For the good searcher, a decrease in the entitlement shifts the contract uniformly downwards (figures 6-9), as can be expected.

We can also see from figures 6 to 9 that for the bad searcher the MH effect prevails for high entitlement bounds $V$ and thus his optimal contract falls as well (figure 6). But as $\underline{V}$ is lowered the AS 
effect becomes more and more important and the benefit profile becomes hump shaped (figure 8) or even rises (figure 9).

It is interesting to note that at the same time, according to table 1 (compare also table 3 and 4), the information rent for agent $\mathrm{G}$ is increasing as $\underline{V}$ falls. We infer that the distortion of contract b away from the contract for B in a pure moral hazard environment (compare Corollary 3.9) increases as $\underline{V}$ is lowered. So broadly speaking we can say that the rent extraction/search efficiency trade-off is more severe for low than for high values of $\underline{V}$. How can this be explained?

Let us first briefly mention a mechanism that reinforces the MH effect as $\underline{V}$ increases but cannot fully account for the observed changes in benefit profiles. As can be seen from table 2, the elasticity of the probability of remaining unemployed $\sigma_{\pi}^{i}(z)$ with respect to the UI benefits are increasing in the benefit level.

This means that at higher levels of utility a reduction of future benefits has a greater effect on the search effort and thus the reemployment chances of the unemployed agent. Hence the $\mathrm{MH}$ effect is more likely to matter at higher levels of utility, as we see in the figures. However, the tables show that the elasticities $\sigma_{\pi}^{i}(z)$ alter only slightly as the utility $\underline{V}$ is lowered. Thus this "elasticity effect" alone cannot explain the changes we observe in the result of the comparative static exercise.

The main force driving the result is the convexity of the cost function, i.e., the fact that the marginal costs of providing a certain utility increases in the level of utility. Put in terms of the agents' utility function we can say that a reduction of benefits hurts an agent disproportionately badly at a low level of utility. So in particular, a cost neutral shift of a benefit scheme from a flat to a bended profile comes at a greater relative loss of utility at low utility levels than at high utility levels. Loosely speaking, consumption smoothing is more important at low levels of utility than at high levels.

Now we look at contract b. Its utility $V^{b, B}$ for agent B is fixed at $\underline{V}$, and so we concentrate on its value $V^{b, G}$ for $\mathrm{G}$. As we have pointed out above, the rent extraction/search efficiency trade-off is reflected by the AS and the MH effect: The slope of contract b is upward sloping in a pure AS and downward sloping in a pure 
$\mathrm{MH}$ environment. Assume for a moment that the elasticity of the probability of remaining unemployed $\sigma_{\pi}^{i}(z)$, that determines the importance of the $\mathrm{MH}$ effect, remains constant along shifts of the entitlements (actually we have seen that the increase of $\sigma_{\pi}^{i}(z)$ in $z$ even reinforces the results). In other words: We assume for a moment that the search efficiency of $B$ is equal across levels of utility.

Now according to what we have said about the preferences for flat versus bended contracts of agents with concave utility functions, we deduce that agent $\mathrm{G}$ would prefer to have his consumption smoothed more strongly at low than at high levels of benefits. Since his own contract $\mathrm{g}$ is falling, the (flatter) contract $\mathrm{b}$ becomes more attractive to him as the utility level is reduced. So in order to ensure that the agents are separated, in equilibrium the principal grants $\mathrm{G}$ a higher information rent (in relative terms, i.e. as a fraction of his entitlement) and accepts a stronger distortion of contract b from the pure moral hazard contract for B as $\underline{V}$ is lowered (recall that we hold the $\mathrm{MH}$ effect fixed for the moment). The distorted (upward sloping) contract b is then relatively less attractive for $\mathrm{G}$ than for B because of the Spence-Mirrlees property, as explained above.

The share of type B agents $q$ Figures 10, 9 and 11 depict the optimal contracts at the entitlement bound $\underline{V}=-35$ (Certainty equivalent: $49.03 \%$ ) for $q=0.2,0.5$ and 0.8 respectively. As the share of the bad searchers $q$ increases, we see a clear shift of contract $\mathrm{b}$ dominated by the AS effect to one dominated by the $\mathrm{MH}$ effect. Furthermore, the contract for the good searcher is uniformly shifted upwards. Moreover, from table 1 we can see that his information rent rises.

We can explain these observations as follows: With a small proportion of type $\mathrm{B}$ agents, the principal concentrates on lowering the costs of contract g. He does so by pushing down the information rent of agent $\mathrm{G}$, thus heavily distorting the value of contract $b$ for agent $G$ (recall that the incentive constraint for the good searcher binds, $\left.V^{b, G}=V^{g, G}\right)$. Since the value of contract b for agent $\mathrm{B}$ is fixed at $\underline{V}$, the value $V^{b, G}$ can only be lowered by steeply raising the benefit scheme $b$. This is true because of the 
Information Rents

\begin{tabular}{c|c|rrr}
$\underline{V}$ & Certainty Equivalent & $\theta_{B}=0.004$ & $\theta_{B}=0.007$ & $\theta_{B}=0.010$ \\
\hline \hline-35 & 49.03 & $20.6 \%$ & $18.1 \%$ & $14.2 \%$ \\
-30 & 55.21 & $14.3 \%$ & $13.9 \%$ & $10.9 \%$ \\
-25 & 61.76 & $9.7 \%$ & $10.5 \%$ & $8.2 \%$ \\
-20 & 68.67 & $6.6 \%$ & $7.2 \%$ & $6.2 \%$
\end{tabular}

(rents expressed as percentage increase over minimal entitlement)

Table 3: Information Rents obtained by agent $\mathrm{G}$

Spence-Mirrlees property.

With an increasing proportion of type B agents, reducing the costs arising from their contract predominates the principal's problem and the issue of paying an information rent to type $G$ agents looses importance. In order to keep the costs of contract b low, the principal prefers to avoid a large distortion of $V^{b, G}$ away from its first best and consequently accepts a higher information rent paid to type $\mathrm{G}$ agents.

Economically speaking, with a high proportion of type $\mathrm{G}$ agents, the principal wants to curb the information rent, whereas with a high proportion of type $\mathrm{B}$ agents, the principal wants to ensure the search efficiency of type B agents.

Agent B's unemployment probability parameter $\theta_{B}$ The parameter $\theta_{B}$ determines the search capacity of agent $\mathrm{B}$ : The higher it is, the lower is his probability of remaining unemployed, given the same search effort $a$. We have looked at the case where the agents become more similar in their search technology, i.e. $\theta_{B}$ increases while $\theta_{G}$ is held fix. In our analysis, we have therefore kept agent G's search parameter at $\theta_{G}=0.017$ and have looked at the cases of $\theta_{B}=0.004, \theta_{B}=0.007$ and $\theta_{B}=0.010$. The latter correspond to an escape rate in autarky of $15.6 \%, 22.7$ and $27.7 \%$ per month respectively. Figures 12,8 and 13 show the optimal contracts at a utility level of $\underline{V}=-30$ (certainty equivalent: $55.21 \%$ ). From these figures we see that the AS effect prevails for low values of $\theta_{B}$, whereas for high values the $\mathrm{MH}$ effect is dominant. 
Information Rents

\begin{tabular}{c|rrr} 
Certainty Equivalent & $\alpha=1.5$ & $\alpha=2$ & $\alpha=2.5$ \\
\hline \hline 49.03 & $27.3 \%$ & $18.1 \%$ & $10.4 \%$ \\
55.21 & $21.5 \%$ & $13.9 \%$ & $8.2 \%$ \\
61.76 & $17.1 \%$ & $10.5 \%$ & $5.8 \%$ \\
68.67 & $13.7 \%$ & $7.2 \%$ & $3.7 \%$
\end{tabular}

(rents expressed as percentage increase over minimal entitlement)

Table 4: Information Rents obtained by agent $\mathrm{G}$

For the good searcher an increase in $\theta_{B}$ shifts his benefit scheme downwards. At the same time an increase in $\theta_{B}$ implies for the bad searcher that the AS aspect looses importance and the $\mathrm{MH}$ becomes increasingly important. One reason for this can be seen from table 2 . The elasticity of the probability of remaining unemployed is increased considerably as $\theta_{B}$ rises. As explained in the paragraph on $\underline{V}$, this means that the search incentives for agent $\mathrm{B}$ are enhanced.

Risk aversion $\frac{\alpha-1}{\alpha}$ The exponent in the cost function $\alpha$ determines the risk aversion of both agents, i.e., the coefficient of relative risk aversion is $\frac{\alpha-1}{\alpha}$. In this paragraph we look at the impact of a change in risk aversion on the optimal contracts and the information rents for type G.The results for the latter two are shown in table 4. As is apparent from the tables we compare levels of certainty equivalents of the unemployment entitlement bounds rather than levels of utility (which would of course make no sense). All other parameters are identical.

Figures 14, 6 and 15 show the optimal contracts at a replacement rate of $68.67 \%$. In these figures we make two observations. First, the contract for type B, that is dominated by the MH effect at $\alpha=1.5$, becomes smoother as $\alpha$ increases. Furthermore it slightly shifts upwards. Second, we can see that the falling contract for $\mathrm{G}$ is shifted upwards as $\alpha$ increases. At the same time his information rent falls (see table 4). 
The economic explanation for these two phenomena is straightforward. In our model the coefficient of relative risk aversion is identical to the inverse of the intertemporal elasticity of substitution of consumption, i.e., more risk averse agents have a stronger preference for intertemporal consumption smoothing. Put the other way round: The marginal costs of guaranteeing a certain level of instantaneous utility rise as relative risk aversion rises, so - given that the principal guarantees a specified total expected lifetime utility - falling and rising benefit schemes become relatively more expensive as compared to flat ones. Therefore contracts tend to become flatter as $\alpha$ increases.

For agent $\mathrm{G}$ the contract shifts upwards because his contract is falling. Therefore, as risk aversion increases, he needs to be compensated by higher benefits in order to achieve a given certainty equivalent. This also explains the fall in his information rent. Since his contract becomes disproportionately more expensive in terms of benefits as $\alpha$ increases (recall the convexity of the cost function), the UI agency wants to save information rent. This mechanism resembles the fall of the information rent for $G$ as $\underline{V}$ increases (see above).

\section{Extensions}

In this section we want to discuss a number of possible extensions of our model. A first extension concerns the (minimal) entitlement $\underline{V}$ for the unemployed: It could be type-dependent. Second, we show how to integrate the UI taxes paid after reemployment introduced by HN. Third, we discuss the limit of the time horizon $T$ going to infinity.

Type Dependent Entitlement Constraint The entitlement constraints in our model correspond technically to participation constraints in adverse selection models analyzed in classical contract theory. The case of type-dependent reservation utility in participation constraints has been widely discussed in that context, the most general work being the article by ?. For our purpose, we will refer to Section 3.3.1 of Laffont and Martimort (2002). They 
distinguish between five cases, characterized by different combinations of participation and incentive constraints binding at the solution.

Clearly - given Proposition 3.8 - only the case where the minimal entitlement of agent $\mathrm{G}, \underline{V}_{G}$, is greater than the one of agent B, $\underline{V}_{B}$, i. e. $\underline{V}_{G}>\underline{V}_{B}$, is interesting ${ }^{13}$. Otherwise the solution is identical to the one we have found there. This is also the case for $\underline{V}_{G}$ being only slightly greater than $\underline{V}_{B}$; more precisely we can raise $\underline{V}_{G}$ up to the value of $V^{g, G}$ (greater than $\underline{V}_{B}$ by Corollary 3.10) in Proposition 3.8 without changing the results. This corresponds to the case 1 of Laffont and Martimort (2002). Note, however, that we do not know whether the incentive constraint of the bad searcher B is slack or binding at the solution (although numerically we found that it was slack). In the first case - that has been the resulting from our simulations - for $\underline{V}_{G}=V^{G, g}+\epsilon$ with some $\epsilon>0$ we would have a situation as in their case 2 (with both entitlement constraints and the good searchers incentive constraint binding). In the second case we would face either their case 4 (both entitlement constraints binding and type B's incentive constraint binding) or case 5 (type G's entitlement type B's incentive constraint binding). In principle, as the wedge between $\underline{V}_{B}$ and $\underline{V}_{G}$ widens, we expect the entitlement constraint of $\mathrm{G}$ to be binding at the solution, his contract becoming more and more attractive for type B. In the case of a very large gap, we will have to compensate $\mathrm{B}$ with an information rent for not choosing the contract of type G, so it is G's entitlement constraint and B's incentive constraint that have to be binding at the solution (case 5 of Laffont and Martimort (2002)). So the situation is reversed with respect to Proposition 3.8: The contract for agent B takes the same form as in the pure moral hazard environment, the contract for $\mathrm{G}$ will be distorted. Note that it will be distorted downwards to make it less attractive for agent $\mathrm{B}$.

For the values of the wedge $\left(\underline{V}_{G}-\underline{V}_{B}\right)$ in between we should expect solutions according to case 2 to 4 of Laffont and Martimort (2002). A precise description of the solution in analytical terms,

\footnotetext{
${ }^{13} \mathrm{As}$ in Proposition 3.8 we will assume that that $\underline{V}_{i}$ with $i=B, G$ are not too small
} 
i.e. a statement about constraints being binding or slack for certain ranges of parameter values, will probably be untractable due to the highly non-linear dependence of the entitlements on basic parameters of the problem. However, a numerical analysis should be straightforward, because it amounts to a direct comparison of solutions of minimization problems with different (binding) constraints.

Taxes on wage Shavell and Weiss (1979) analyze the optimal allocation of UI benefits with one representative agent, $\mathrm{HN}$ add taxes on labor income after reemployment to this analysis. They make the simplifying assumption that the tax rate is fixed for the rest of the life the moment agents have gained reemployment. In our model we have followed Shavell and Weiss (1979), technically, however, the extension to the framework of $\mathrm{HN}$ is straightforward. By taxing (or subsidizing) labor income the principal can completely control the agent's consumption when he is employed. The additional ability of the principal to tax the agent can thus be captured by the introduction of entitlements while employed, $W_{t}$, that take the role of additional state variables, and a value function for transfers, that corresponds to the cost function in the recursive formulation of the UI contract in the model by Shavell and Weiss (1979). In our model we would have to introduce two additional state variables for each contract $i, W^{i, B}$ and $W^{i, G}$. These state variables state the entitlement utility of an employed agent under contract $i$. Given the wage rate, the transfer to the principal can be calculated directly.

Although this extension does not seem to be complicated at first glance, there is a problem. In the recursive formulation of contract $\mathrm{b}$ (and $\mathrm{g}$ likewise), given by Proposition 3.2, the additional state variables enter into the cost functions of the principal and -as choice variables- into the minimization problem in the backward induction. While the value of the transfers after the agent's reemployment are easily calculated, the approximation of the cost function poses a serious numerical problem. This is because it takes now four variables, and the curse of dimensionality becomes a serious problem. 
Infinite Time Horizon The finite time horizon of our model permitted us to calculate the correspondence of feasible entitlements $\Gamma_{t}\left(V_{t}^{B}, V_{t}^{G}\right)$ by backward induction in our simulation of optimal contracts. In designing the algorithm we relied in particular on the properties of the set of jointly feasible entitlements, the sets being compact, connected and contractible.

As in Proposition 3.2, the recursive formulation of an optimal contract given some $\left(V^{B}, V^{G}\right)$ is straightforward in the case of an infinite time horizon:

$$
C^{B}\left(V^{B}, V^{G}\right)=\min _{\left\{z, \hat{V}^{B}, \hat{V}^{G}\right\} \in \Gamma\left(V^{B}, V^{G}\right)} c(z)+\beta p_{B}\left(a^{B}\right) C^{B}\left(\hat{V}^{B}, \hat{V}^{G}\right)
$$

subject to:

Law of motion for contract b (LOM)

$$
\begin{aligned}
& z-a^{B}+\beta\left[p_{B}\left(a^{B}\right) \hat{V}^{B}+\left(1-p_{B}\left(a^{B}\right)\right) W\right]=V^{B} \\
& z-a^{G}+\beta\left[p_{G}\left(a^{G}\right) \hat{V}^{G}+\left(1-p_{G}\left(a^{G}\right)\right) W\right]=V^{G}
\end{aligned}
$$

Choice of effort in contract b (CE)

$$
\begin{aligned}
& a^{B}=\operatorname{argmax}_{a} z-a+\beta\left[p_{B}(a) \hat{V}^{B}+\left(1-p_{B}(a)\right) W\right] \\
& a^{G}=\operatorname{argmax}_{a} z-a+\beta\left[p_{G}(a) \hat{V}^{G}+\left(1-p_{G}(a)\right) W\right] .
\end{aligned}
$$

This is a Bellman equation, where pairs of entitlements today $\left(V^{B}, V^{G}\right)$ are the state variables and benefit today and promises for tomorrow are the choice variables $\left(z, \hat{V}^{B}, \hat{V}^{G}\right)$. The value of lifetime work is denoted by $W$. Once the cost functions are determined, the full adverse selection problem can be stated as in Proposition 3.8. Before this can be done, an important question arises: How to characterize the correspondence $\Gamma\left(V^{B}, V^{G}\right)$ ?

We conjecture that the correspondence $\Gamma\left(V^{B}, V^{G}\right)$ has the same properties as $\Gamma_{t}\left(V_{t}^{B}, V_{t}^{G}\right)$ in Proposition 3.4. In particular, the set of jointly feasible state variables $\Omega=\left\{\left(V^{B}, V^{G}\right) \mid \Gamma\left(V^{B}, V^{G}\right) \neq \emptyset\right\}$ will be compact, connected and contractible. Since the choice problem of the principal can be reduced in the same way to the one-dimensional problem of choosing $a^{G}$ using the LOM as before, these properties of the set of jointly feasible state variables would guarantee that the functional form of the correspondence does not 
change when the time horizon $T$ goes to infinity.

Here we outline a proof. It is based on the idea that the set of jointly feasible entitlements in the case of an infinite time horizon is the inverse limit of the sets $\Omega_{t}=\left\{\left(V_{t}^{B}, V_{t}^{G}\right) \mid \Gamma_{t}\left(V_{t}^{B}, V_{t}^{G}\right) \neq \emptyset\right\}$ of jointly feasible entitlements in the finite dimensional case (with the topology being -of course- the topology of the two dimensional real space):

$$
\Omega=\lim _{\leftarrow} \Omega_{t} .
$$

At the same time, given a lower bound on the one period benefit $\underline{z}$, we know that $\Omega$ must be bounded. Thus we obtain compactness. To show that $\Omega$ is contractible, we need some homotopy theory. We apply a theorem giving a hint about the first homotopy group of $\Omega, \pi_{1}(\Omega)$ (compare ?, p. 254):

$$
0 \rightarrow \lim _{\leftarrow} \pi_{2}\left(\Omega_{t}\right) \rightarrow \pi_{1}(\Omega) \rightarrow \lim _{\leftarrow} \pi_{1}\left(\Omega_{t}\right) \rightarrow 0
$$

Moreover, due to the relation between $\Gamma_{t}$ and $\Gamma_{t+1}$, the natural mapping $\Omega_{t} \rightarrow \Omega_{t+1}$ is a fiber bundle. Therefore we conclude that both $\lim _{\leftarrow} \pi_{2}\left(\Omega_{t}\right)$ and $\lim _{\leftarrow} \pi_{1}\left(\Omega_{t}\right)$ must vanish, yielding the contractibility of $\Omega$.

We thus believe that Proposition 3.4 is a technical result that matters for dynamic programming with infinite time horizons, i.e. for economies of the type considered in APS, Atkeson and Lucas (1992), Chang (1998). However, we leave a precise generalization to future research.

Acknowledgement We would like to thank Michael Reiter for invaluable support. We thank seminar participants at the ZEI b breakfast seminar, the BGSE Workshop (Bonn), VII. Workshop on Dynamic Macroeconomics of the University of Vigo 2003, the Mannheim Brown Bag Seminar on Applied Economics, the Jamboree of the EDP at the LSE, the Macroeconomics Brown Bag Seminar of the Humboldt University and the Macro Workshop at UPF for helpful comments. Tim Mennel gratefully acknowledges financial assistance by the Marie-Curie-Fellowship program. 


\section{References}

Abreu, D., D. Pearce, and E. Stacchetti, 1990, Toward a theory of discounted repeated games with imperfect monitoring, Econometrica 58.

Atkeson, A., and R. E. Lucas, 1992, On efficient distribution with private information, Review of Economic Studies 59.

Atkinson, A. B., and J. Micklewright, 1991, Unemployment compensation and labor market transitions: A critical review, Journal of Economic Literature 29.

Chang, R., 1998, Credible monetary policy in infinite horizon model: Recursive approaches, Journal of Economic Theory 81.

Fernandes, A., and C. Phelan, 2000, A recursive formulation for repeated agency with history dependence, Journal of Economic Theory 99.

Fredriksson, P., and B. Holmlund, 2003, Improving incentives in unemployment insurance, Working paper Uppsala University.

Hopenhayn, H. A., and J. P. Nicolini, 1997, Optimal unemployment insurance, Journal of Political Economy 105.

, 2001, Heterogeity and optimal unemployment insurance, in N. Lustig, ed.: Shielding the Poor (Brookings Institution Press).

Judd, K. L., 1998, Numerical Methods in Economics (MIT Press).

Karni, E., 1999, Optimal unemployment insurance: A survey, Southern Economic Journal 66.

Laffont, J. J., and D. Martimort, 2002, The Theory of Incentives (Princeton University Press).

Meyer, B., 1990, Unemployment insurance and unemployment spells, Econometrica 58. 
_ 1995, Lessons from the u.s. unemployment insurance experiments, Journal of Economic Literature 23.

Mortensen, D. T., 1983, A welfare analysis of unemployment insurance: Variations on second best themes, Carnegie-Rochester Conference Series 19.

Pavoni, N., 2000, Three essays on social insurance, Ph.D. thesis Universitat Pompeu Fabra.

Rothschild, M., and J. Stiglitz, 1976, Equilibrium in competitive insurance markets: An essay on the economics of imperfect information, Quarterly Journal of Economics 90.

Shavell, S., and L. Weiss, 1979, The optimal payment of unemployment insurance benefits over time, Journal of Political Economy 87.

Spear, S., and S. Srivastava, 1987, On repeated moral hazard with discounting, Review of Economic Studies 54.

Thomas, J., and T. Worrall, 1990, Income fluctuation and asymmetric information: An example of a repeated principal/agent problem, Journal of Economic Theory 51.

Wang, C., and S. D. Williamson, 2002, Moral hazard, optimal unemployment insurance and experience rating, Journal of Monetary Economics 49. 


\section{Appendix}

\section{Proof of Lemma 2.7}

Proof. Firstly, we prove that $p_{B}\left(a_{t}^{B}\right)>p_{G}\left(a_{t}^{G}\right)$ ensues from the Spence-Mirrlees-Property 7. We calculate

$$
\frac{\partial V_{t}^{i}}{\partial z_{t+1}}=\beta p_{i}\left(a_{t}^{i}\right) \frac{\partial V_{t+1}^{i}}{\partial z_{t+1}}=\beta p_{i}\left(a_{t}^{i}\right)
$$

where we use the Envelope Theorem. The assertion now follows immediately.

Secondly, assume that $p_{B}\left(a_{t}^{B}\right)>p_{G}\left(a_{t}^{G}\right)$ holds. In the case where $s=1$, the Spence-Mirrlees property follows from what we have shown above. So let $s>1$. Then

$$
\frac{\partial V_{t}^{B}}{\partial z_{t+s}}-\frac{\partial V_{t}^{G}}{\partial z_{t+s}}=\beta p_{B} \frac{\partial V_{t+1}^{B}}{\partial z_{t+s}}-\beta p_{G} \frac{\partial V_{t+1}^{G}}{\partial z_{t+s}}
$$

where we have used the Envelope Theorem once more. The assertion follows by induction.

\section{Proof of Corollary 3.9}

Proof. The first assertion is point 2 in the proof of 3.8. To see the second assertion, note that, given that $V^{g, B}$ is chosen optimally for each value of $V^{g, G}$, the cost function of contract $g$ is strictly increasing in $V^{g, G}$. Moreover, in a full information optimum (i.e. the pure moral hazard case for both contracts) the optimal $V^{b, G}$ (optimal with respect to $V^{b, B}=\underline{V}$ ) can be characterized by a first order condition. We thus obtain a first order reduction of costs for contract $\mathrm{g}$ by lowering $V^{g, G}=V^{b, G}$ (constraint 19 is binding!) below the value of $V^{b, G}$ in a full information optimum, whereas there is only a second order increase in costs for contract b.

\section{Proof of Corollary 3.10}

Proof. The fact that $V^{g, G}>\underline{V}$ has been proved in Proposition 3.8; so we look at the second assertion. In our framework, we can recover the Shavell-Weiss contracts (i.e. the contracts from the pure moral hazard environment) at a given level of entitlement 
$V^{i, i}$ by solving $(i \neq j)$ :

$$
\begin{array}{ll} 
& \min _{V^{i, j}} C_{1}^{i}\left(V^{i, i}, V^{i, j}\right) \\
\text { s.t. } & L O M, M H-I C
\end{array}
$$

and applying forward induction afterwards. This is because by minimizing the costs of contract $i$ with respect to its value for agent $j$, we just neglect the impact of this value for the optimal contract.

Now, if our objective function is optimized without further restriction, we recover the optimal contract from the pure moral hazard environment, because the value $V^{g, B}$ of contract $g$ for type $\mathrm{B}$ does not appear in the cost function of contract $\mathrm{b}$.

\section{Proof of Theorem 3.1}

Proof. It is clear that there is a solution to the principal's problem. Let assumption 2.8 hold. We prove the second assertion by contradiction: Assume that the principal's problem is solved by one contract $\mathrm{p},\left\{z_{1}^{P}, \ldots, z_{T-1}^{P}, z_{T}^{P}\right\}$, for both agents, that generates a total expected utility of $V_{t}^{B}$ and $V_{t}^{G}$ in period $t$ for agent B and $\mathrm{G}$ respectively.

We have a look at the "first best" solutions for the last two periods that generate the same utilities $V_{T-1}^{B}$ and $V_{T-1}^{G}$ as p. As "first best" solution, we refer to the solution of the pure moral hazard problem as considered by Shavell and Weiss (1979), i.e. the problem of guaranteeing agent $i$ a utility of $V_{T-1}^{i}$ at lowest cost. This is stated as follows:

$$
\begin{gathered}
\min _{z_{T-1}^{i}, z_{T}^{i}} c\left(z_{T-1}^{i}\right)+\beta p_{i}\left(a^{i}\right) c\left(z_{T}^{i}\right) \\
\text { s.t. } \quad V_{T-1}^{i}=z_{T-1}^{i}-a^{i}+\beta\left[p\left(a^{i}\right) z_{T}^{i}+\left(1-p\left(a^{i}\right)\right) u(w)\right] \\
1=\beta p_{i}^{\prime}\left(a^{i}\right)\left[z_{T}^{i}-u(w)\right]
\end{gathered}
$$

This is the two period cost minimization problem (the principal's problem in this framework) in the case of agent $i$, subject to the promise keeping constraint and the first order condition of the agent's problem, determining the choice of effort $a^{i}$. Plugging the entitlement constraint into the objective function and making use of the envelop theorem, we calculate the following first order conditions for the principal with respect to $z_{T}$ (we abbreviate $p_{i}=$ 
$\left.p_{i}\left(a^{i}\right)\right)$

$$
\begin{aligned}
& c^{\prime}\left(z_{T-1}^{B}\right)=-\frac{\left(p_{B}^{\prime}\right)^{3}}{p_{B} p_{B}^{\prime \prime}} c\left(z_{T}^{B}\right)+c^{\prime}\left(z_{T}^{B}\right) \\
& c^{\prime}\left(z_{T-1}^{G}\right)=-\frac{\left(p_{G}^{\prime}\right)^{3}}{p_{G} p_{G}^{\prime \prime}} c\left(z_{T}^{B}\right)+c^{\prime}\left(z_{T}^{B}\right) .
\end{aligned}
$$

The factor of the cost function on the right-hand side is

$$
-\frac{\left(p_{i}^{\prime}\right)^{3}}{p_{i} p_{i}^{\prime \prime}}=\frac{1}{\pi_{i}\left(z_{T}\right)} \frac{\partial \pi_{i}\left(z_{T}\right)}{\partial z_{T}},
$$

and so we see that the RHS is identical to the relative expected marginal cost. By condition 2.8, part 1, we know that factor of the cost function is higher for agent $\mathrm{G}$ than for agent $\mathrm{B}$ for a given $z_{T}$. By its second part we know that this has to hold in equilibrium, too, and so the RHS is greater for agent $\mathrm{G}$ than for agent B. ${ }^{14} \mathrm{We}$ may therefore deduce that the Shavell-Weiss contract of agent $B$ is flatter than its counterpart for agent G, where we define "flatter" in the following sense:

$$
\frac{z_{T-1}^{G}}{z_{T}^{G}}>\frac{z_{T-1}^{B}}{z_{T}^{B}} .
$$

In the following we will dicuss the last two periods of the pooling contract only and show that it cannot be optimal to offer it to both agents. We will refer to the first best solutions as Shavell-Weiss (SW) contracts.

First, suppose that the pooling contract $\mathrm{p}$ is flatter than the SW contract of agent G. Then the principal can offer $p$ and a second contract g' that is identical to contract $\mathrm{p}$ except for the last two periods, where $z_{T-1}^{P}$ and $z_{T}^{P}$ are substituted by $z_{T-1}^{G}$ and $z_{T-1}^{G}$ from the SW contract. This is incentive compatible: Agent $\mathrm{G}$ is indifferent between $\mathrm{p}$ and g' by construction. Suppose that agent B (weakly) preferred g' over p. Then for period 1 to $T-2$, he can exert the same effort $a_{1}^{g}$ to $a_{T-2}^{g}$ (i.e. that he chooses in the case of contract g') when facing contract $p$, and thus the stochastically discounted utility from the benefits $z_{1}$ to $z_{T-2}$ is identical for both contracts. In the last two periods, in contrast, agent B -exerting

\footnotetext{
${ }^{14}$ Note that we could weaken condition 2.8: To ensure that the RHS of G is higher than the RHS of B it is sufficient to assume that the relative marginal probability of remaining unemployed $\frac{1}{\pi_{i}(z)} \frac{\partial \pi_{i}(z)}{\partial z}$ is higher for agent $\mathrm{G}$ than for agent $B$.
} 
effort optimally- gains a higher utility from the flatter contract $\mathrm{p}$ than from contract g' because of the Spence-Mirrlees property (cf. Lemma 2.7). So agent B cannot prefer g' over p. Offering the two contracts $\mathrm{p}$ and g' is also cheaper for the principal, because g' is the (unique) cost-optimizing contract for agent $\mathrm{G}$ during the last two periods. Contradiction.

Second, suppose that the pooling contract $\mathrm{p}$ is identical to or steeper than the SW contract of agent G. The principal then offers $\mathrm{p}$ and a second contract b' that is identical to contract $\mathrm{p}$ with $z_{T-1}^{P}$ and $z_{T}^{P}$ substituted by $z_{T-1}^{B}$ and $z_{T}^{B}$ from the SW contract. Since the SW contract of agent $B$ is flatter than the SW contract of agent $\mathrm{G}$, as we have seen, we can infer the contradiction in the same way as in the first case.

\section{Proof of Proposition 3.4}

Proof. In order to simplify the proof we introduce a normalization: The utility from consuming the wage $w$ is set to zero. Thus, all $W_{t}$ become zero, too, and the entitlement utilities of the unemployed agents take non-positive values. Note that the lower bounds for the entitlements, stemming from the lower bound on the benefit utility $\underline{z}$, thus shift downward each period along the backward induction.

First, we look at the agents' problem. Recall it takes the form

$$
V_{t}^{i}=\max _{a} z_{t}-a+\beta\left[p_{i}(a) V_{t+1}^{i}+\left(1-p_{i}(a)\right) W_{t+1}\right] .
$$

Given our normalization we obtain the following first order condition at an interior solution:

$$
p_{i}^{\prime}\left(a_{t}^{i}\right)=\frac{1}{\beta V_{t+1}^{i}}
$$

By the Inada condition in Condition 2.4 we assure that the interior solution always applies.

The Case of $\mathbf{t}=\mathbf{T}-\mathbf{1}$ We start with the case of $\Gamma_{t}\left(V_{t}^{B}, V_{t}^{G}\right)$ with $t=T-1$. Mathematically speaking, the next-to-last period is different from the previous ones in that there is an additional constraint on the choice variables $V_{T}^{i}$ : The boundary conditions 12,13 , namely $V_{T}^{B}=V_{T}^{G}=z_{T}$. This is the very reason why, given the pair of state variables $\left(V_{T-1}^{B}, V_{T-1}^{G}\right)$, there is only one choice left for the principal. First, let's look at the Law of Motion (LOM) for 
the state variables $V_{T-1}^{B}$ and $V_{T-1}^{G}$ :

$$
\begin{aligned}
& z_{T-1}-a_{T-1}^{B}+\beta p_{B}\left(a_{T-1}^{B}\right) V_{T}^{B}=V_{T-1}^{B}, \\
& z_{T-1}-a_{T-1}^{G}+\beta p_{G}\left(a_{T-1}^{G}\right) V_{T}^{G}=V_{T-1}^{G},
\end{aligned}
$$

where we will drop the time index from the effort variables $a_{T-1}^{i}$. In the following, we will denote the difference between the entitlements of the agents by:

$$
\Delta_{t}:=V_{t}^{G}-V_{t}^{B}
$$

With this new notation and remembering both our normalization and $V_{T}^{i}=z_{T}$, we solve the LOM for $z_{T-1}$, equalize both equations and solve for $\Delta_{T-1}$ :

$$
\Delta_{T-1}=a^{B}-a^{G}+\beta p_{G}\left(a^{G}\right) z_{T}-\beta p_{B}\left(a^{B}\right) z_{T}
$$

We want to further simplify equation 27 . In the next to last period, the first order condition of the agents' problem 25 takes the following form

$$
p_{B}^{\prime}\left(a^{B}\right)=p_{G}^{\prime}\left(a^{G}\right)=\frac{1}{\beta z_{T}} .
$$

Again by Condition 2.4, the $p_{i}^{\prime}$ are strictly increasing functions

$$
p_{i}^{\prime}:(0, \infty) \longrightarrow(-\infty, 0) .
$$

Remark .1 Given Condition 2.3, the principal will never promise an entitlement above $W_{t}(=0$ under our normalization $)$, since at $W_{t}$ the agents stop searching (i.e. $a_{t}^{i}=0$ ) and their probability of remaining unemployed becomes $p_{i}\left(a_{t}^{i}\right)=1$.

Thus in particular $V_{T}^{i}=z_{T}<0$.

¿From this we deduce that the $p_{i}^{\prime}$ are one-to-one and onto. Therefore the following function $\gamma\left(a^{G}\right)$ is well defined:

$$
\gamma\left(a^{G}\right):=\left(p_{B}^{\prime}\right)^{-1} \circ p_{G}^{\prime}\left(a^{G}\right) .
$$

Now we have everything at hand to define $\Delta_{T-1}$ as a function of $a^{G}$ :

$$
\Delta_{T-1}\left(a^{G}\right)=\gamma\left(a^{G}\right)-a^{G}+\frac{p_{G}\left(a^{G}\right)-p_{B}\left(\gamma\left(a^{G}\right)\right)}{p_{G}^{\prime}\left(a^{G}\right)}
$$

In order to show point 3 of Proposition 3.4, we have to show that $\Delta_{T-1}($.$) is invertible.$ 
We do so by proving:

$$
\Delta_{T-1}^{\prime}\left(a^{G}\right)>0
$$

Using the agents' first order condition 25 and

$$
\gamma^{\prime}\left(a^{G}\right)=\frac{p_{G}^{\prime \prime}\left(a^{G}\right)}{p_{B}^{\prime \prime}\left(\gamma\left(a^{G}\right)\right)}
$$

we calculate

$$
\Delta_{T-1}^{\prime}\left(a^{G}\right)=\left[p_{B}\left(\gamma\left(a^{G}\right)\right)-p_{G}\left(a^{G}\right)\right] \frac{p_{G}^{\prime \prime}\left(a^{G}\right)}{\left(p_{G}^{\prime}\left(a^{G}\right)\right)^{2}} .
$$

By Condition 2.4 we know that $p_{G}^{\prime \prime}()>$.0 , and since $p_{B}\left(\gamma\left(a^{G}\right)\right)>$ $p_{G}\left(a^{G}\right)$ by Condition 2.6 assertion 30 follows.

Finally we observe that -again by Condition 2.4-

$$
\lim _{a^{G} \rightarrow 0} \Delta_{T-1}\left(a^{G}\right)=0 .
$$

Together with 30 we deduce that as agent B's entitlement $V_{T-1}^{B}$ approaches agent $\mathrm{G}^{\prime}$ 's one $V_{T-1}^{G}$, the effort of the agent $\mathrm{G} a^{G}$ (as well as the effort of agent $\mathrm{B}$ ) go to zero. Because of 25 this means that the benefit for the last period $z_{T}$ has to converge to zero, i.e. the wage consumption utility.

Summarizing our results so far, we can state the following: Given entitlements $V_{T-1}^{B}$ and $V_{T-1}^{G}$ such that $\Delta_{T-1} \geq 0$, we can find a unique corresponding choice of effort by agent $G a^{G}$ (for the time being, we neglect the lower bound $\underline{z}$ on the benefits $z_{t}$ ). From this we can calculate -uniquely- the choice of effort by agent $\mathrm{B} a^{B}$ and the benefit for the last period $z_{T}$ from equation 25, and the benefit of the next to last period $z_{T-1}$ from LOM. All these functions are differentiable. As $\Delta_{T-1}$ goes to zero, the benefit of the last period $z_{T}$ goes to zero, i.e. the cost of the benefit converges to the wage.

We finally have to look at the set of feasible entitlements $V_{T-1}^{B}$ and $V_{T-1}^{G}$. If $\underline{z}=-\infty$, so $z$ can take any value, we infer from 32 that the upper bound $\bar{V}_{t}^{B}($.$) on V_{t}^{B}$, given $V_{t}^{G}$, is

$$
\bar{V}_{t}^{B}\left(V_{t}^{G}\right)=V_{t}^{G} .
$$

As for the lower bound, we calculate

$$
\underline{V}_{t}^{B}\left(V_{t}^{G}\right)=\lim _{a^{G} \rightarrow \infty} V_{t}^{G}-\Delta_{T-1}\left(a^{G}\right) .
$$


Now let $\underline{z}>-\infty$. Then there is a natural lower bound $\underline{V}_{T-1}^{G}$, namely the stochastically discounted sum of the bounds on $z_{T-1}$ and $z_{T}$. Given $V_{T-1}^{G} \in\left[\underline{V}_{T-1}^{G}, 0\right]$ we now have to prove that there is a lower and an upper bound $\underline{V}_{T-1}^{B}\left(V_{T-1}^{G}\right)$ and $\bar{V}_{T-1}^{B}\left(V_{T-1}^{G}\right)$ on the corresponding feasible $V_{T-1}^{B}$. Because of 25 the lower bound on $z_{T}$ translates into an upper bound $\bar{a}^{G}$ on the corresponding choices of effort of agent G. It is attained with equality. By 26 and 30 we find the lower bound

$$
\underline{V}_{T-1}^{B}\left(V_{T-1}^{G}\right)=V_{T-1}^{G}-\Delta_{T-1}\left(\bar{a}^{G}\right) .
$$

As for the upper bound $\bar{V}_{T-1}^{B}\left(V_{T-1}^{G}\right)$, one can see intuitively that $V_{T-1}^{B}$ is bounded by $V_{T-1}^{G}$ (for a rigorous argument, see point 1 in the proof of 3.8). However, $V_{T-1}^{B}$ does not necessarily attain this bound, because of an additional constraint: $z_{T-1} \geq \underline{z}$. From the LOM and 25 we know

$$
z_{T-1}=V_{T-1}^{G}+a^{G}-\frac{p_{i}\left(a^{G}\right)}{p_{i}^{\prime}\left(a^{G}\right)}
$$

The right hand side is increasing in $a^{G}$, so a lower bound on $z_{T-1}$ implies a lower bound on the effort of the second type, $\underline{a}^{G}$ (note that because of our normalization, the reference points for each period have been shifted downwards). Because of 30 a lower bound on $\Delta_{T-1}$ ensues. Given $V_{T-1}^{G}$, we thus find the upper bound on $V_{T-1}^{B}$ :

$$
\bar{V}_{T-1}^{B}\left(V_{T-1}^{G}\right)=V_{T-1}^{G}-\Delta_{T-1}\left(\underline{a}^{G}\right) .
$$

We see that $V_{T-1}^{B}$ attains $V_{T-1}^{G}$ only if the lower bound $\underline{a}^{G}$ becomes zero (the smallest possible effort). Since $\Delta_{T-1}($.$) is an increasing$ function, we see that all values $V_{T-1}^{B} \in\left[\underline{V}_{T-1}^{B}, \bar{V}_{t-1}^{B}\right]$ are attainable as long as $\bar{a}^{G}>\underline{a}^{G}$. This must be the case for $V_{T-1}^{G} \geq \underline{V}_{T-1}^{G}$, since then there are corresponding benefit values $z_{T-1}, z_{T}$ such that $z_{i} \geq \underline{z}$. Finally, because of the Theorem of the Maximum both $\underline{a}_{T-1}$ and $\bar{a}_{T-1}$ depend continuously on $V_{T-1}^{G}$ and since $\Delta_{T-1}$ is a smooth function, the lower and the upper bound $\underline{V}_{T-1}^{B}$ and $\bar{V}_{T-1}^{B}$ are continuous functions of $V_{T-1}^{G}$.

So for period $T-1$, we have shown that the set of feasible values takes the form stated in the theorem. Note in particular that this set is compact and connected. 
The Case of $\mathbf{t} \leq \mathbf{T}-\mathbf{2}$ In this paragraph, we prove the assertion 1. As we have stated above, the crucial difference between the next to last period and the previous ones is the boundary condition of the last period 12 and 13. Before, for every feasible pair of state variables $\left(V_{T-1}^{B}, V_{T-1}^{G}\right)$-or more precisely, for the difference of these state variables $\Delta_{T-1}$ - there existed one corresponding choice of effort $a_{T-1}^{G}$ that determined all choice variables $\left(z_{T-1}, V_{T}^{B}, V_{T}^{G}\right)$. As we will see below, now to each pair of feasible state variables $\left(V_{t-1}^{B}, V_{t-1}^{G}\right)$ (again, more precisely, to the difference of these state variables $\Delta_{t-1}$ ) there corresponds a line of possible choices of effort $a_{t-1}^{G}$ that parameterizes a compact and connected path of choice variables $\left(z_{t-1}(),. V_{t}^{B}(),. V_{t}^{G}().\right)$.

We have a look at the LOM once more. With the help of the agents' first order condition we transform it into

$$
\begin{aligned}
& z_{t}-a^{B}+\frac{p_{B}\left(a^{B}\right)}{p_{B}^{\prime}\left(a^{B}\right)}=V_{t}^{B}, \\
& z_{t}-a^{G}+\frac{p_{G}\left(a^{G}\right)}{p_{G}^{\prime}\left(a^{G}\right)}=V_{t}^{G},
\end{aligned}
$$

where again we have dropped the time index from $a_{t}^{i}$. This inspires the definition of the following functions $(i=1,2)$

$$
f_{i}\left(a^{i}\right)=a^{i}-\frac{p_{i}\left(a^{i}\right)}{p_{i}^{\prime}\left(a^{i}\right)}
$$

¿From the LOM we can now derive a necessary equation for the choice variables (as represented by the $a^{i} \mathrm{~s}$, replacing the $V_{t}^{i} \mathrm{~s}$ ) to hold:

$$
\Delta_{t}+f_{G}\left(a^{G}\right)=f_{B}\left(a^{B}\right),
$$

where we have used definition 26.

We have a closer look now at $f_{i}$. From

$$
f_{i}^{\prime}=\frac{p_{i} p_{i}^{\prime \prime}}{\left(p_{i}^{\prime}\right)^{2}}>0
$$

we can see that it is a strictly increasing function (bearing in mind Condition 2.4). Moreover we calculate

$$
\begin{aligned}
& \lim _{a^{i} \rightarrow 0} f_{i}\left(a^{i}\right)=0 \\
& \lim _{a^{i} \rightarrow \infty} f_{i}\left(a^{i}\right)=\infty .
\end{aligned}
$$


Now note that there is a natural lower bound $\underline{V}_{t}^{G}$ on each $V_{t}^{G}$, namely the stochastically discounted sum of the $z_{\hat{t}}$ s (where $\hat{t}=$ $t, \ldots, T)$. In the case of $T-1$, we have shown that the set of jointly feasible values $V_{T-1}^{B}, V_{T-1}^{G}$ takes the form stated in the theorem. So let $\Gamma_{t}\left(V_{t}^{B}, V_{t}^{G}\right)$ be non-empty and take the form of a path in the space $\left(z_{t}, V_{t+1}^{B}, V_{t+1}^{G}\right)$ for $V_{t}^{B} \in\left[\underline{V}_{t}^{B}\left(V_{t}^{G}\right), \bar{V}_{t}^{B}\left(V_{t}^{G}\right)\right]$ with $V_{t}^{G} \geq \underline{V}_{t}^{G}$. We have to show first that then $\Gamma_{t-1}\left(V_{t-1}^{B}, V_{t-1}^{G}\right)$ is non-empty for $V_{t-1}^{B} \in\left[\underline{V}_{t-1}^{B}\left(V_{t}^{G}\right), \bar{V}_{t-1}^{B}\left(V_{t}^{G}\right)\right]$ for some continuous functions $\underline{V}_{t-1}^{B}, \bar{V}_{t-1}^{B}$ when $V_{t-1}^{G} \geq \underline{V}_{t-1}^{G}$ and takes the form of a path in $\left(z_{t-1}, V_{t}^{B}, V_{t}^{G}\right)$.

Put differently, we have to ask for which pairs $\left(V_{t-1}^{B}, V_{t-1}^{G}\right)$ there are choice variables $\left(z_{t-1}, V_{t}^{B}, V_{t}^{G}\right)$ that are jointly feasible. By the agents' first order condition 25 we can replace $V_{t}^{B}$ and $V_{t}^{G}$ by the corresponding choices of effort $a_{t-1}^{B}$ and $a_{t-1}^{G}$ (we will drop the time index in the sequel). The effort choices $a^{B}$ and $a^{G}$ have to satisfy equation 33 . Since $\Delta_{t-1} \geq 0$ and by 34,35 and 36 , for all $a^{G} \geq 0$ we can find a corresponding $a^{B} \geq 0$. By the LOM, we can furthermore determine $z_{t-1}$ once $a^{G}$ is given. Thus the number of choice variables $\left(z_{t-1}\left(a^{G}\right), V_{t}^{B}\left(a^{G}\right), V_{t}^{G}\left(a^{G}\right)\right)$ is reduced to the "choice" variable $a^{G}$. All functions are combinations of differentiable functions and thus differentiable. We will call the projection of the triple of choice variables into the two-dimensional space $\left(V_{t}^{B}\left(a^{G}\right), V_{t}^{G}\left(a^{G}\right)\right)$ the curve $\phi_{\Delta_{t-1}}$ parameterized in $a^{G}$.

We have reduced the choice problem to one variable, but which $a^{G}$ correspond to feasible triples $\left(z_{t-1}, V_{t}^{B}, V_{t}^{G}\right)$ ? First we look at the constraint $z_{t-1} \geq \underline{z}$. As in the preceding paragraph, by the LOM

$$
z_{t-1}=V_{t-1}^{G}+f_{G}\left(a^{G}\right)
$$

it translates into a constraint ${ }^{15}$

$$
a^{G} \geq \underline{a}^{G}=\left\{\begin{array}{lll}
f_{G}^{-1}\left(\underline{z}-V_{t-1}^{G}\right) & : & V_{t-1}^{G} \leq \underline{z} \\
0 & : & V_{t-1}^{G}>\underline{z}
\end{array}\right.
$$

Second we have to ask: Which of the pairs of entitlements $\left(V_{t}^{B}\left(a^{G}\right), V_{t}^{G}\left(a^{G}\right)\right)$ are feasible? Well, those for which $\Gamma_{t}\left(V_{t}^{B}, V_{t}^{G}\right)$ is non-empty. In other words: Given $V_{t-1}^{B}$ and $V_{t-1}^{G}$, the set of feasible choices is the intersection of the curve $\phi_{\Delta_{t-1}}$ defined by 33, parameterized in $a^{G}$ with $a^{G} \geq \underline{a}^{G}$, and the set of $\left(V_{t}^{B}, V_{t}^{G}\right)$ with $\Gamma_{t}\left(V_{t}^{B}, V_{t}^{G}\right) \neq \emptyset$. Fig-

\footnotetext{
${ }^{15}$ If $\underline{z}=-\infty$, by our definition there is no limit on $a^{G}$.
} 
ure 5 depicts the intersection for the case of period 4 of 12 in an example from our simulation. The solid lines represent the bounds $\underline{V}_{4}^{B}\left(V_{4}^{G}\right)$ and $\bar{V}_{4}^{B}\left(V_{4}^{G}\right)$, the dotted and the dashed line are curves $\phi_{\Delta_{3}}$ with two different values for $\Delta_{3}$.

Figure 1: Set of jointly feasible entitlements in period 4 of 12

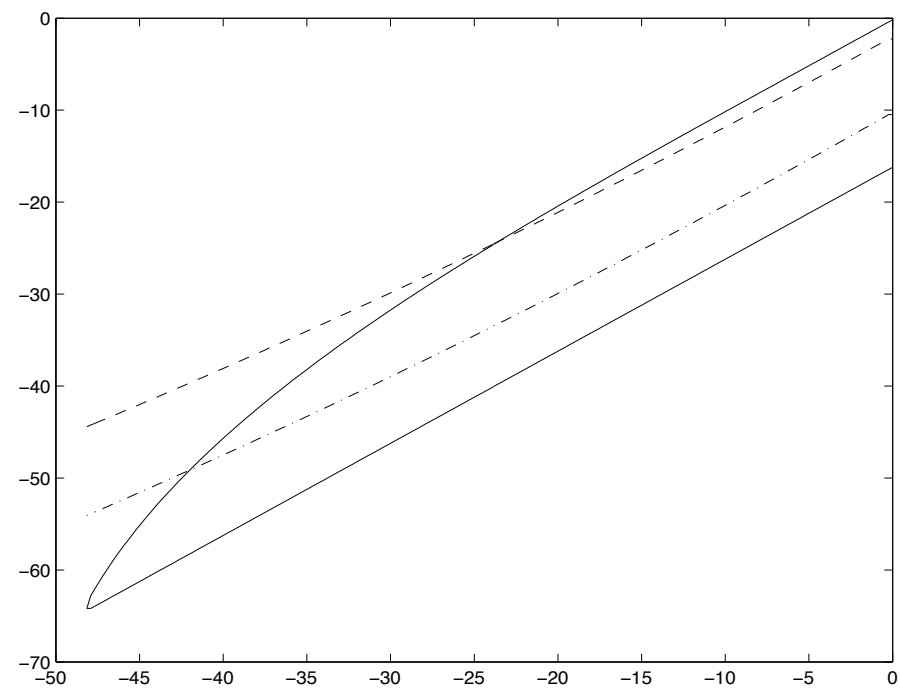

entitlements of type B: type $\mathrm{G}, V_{4}^{B}: V_{4}^{G}$

Two things remain to be shown:

1. we have to show that the set of $\left(V_{t-1}^{B}, V_{t-1}^{G}\right)$, for which the intersection is non-empty, takes itself the form of a set bounded by functions $\underline{V}_{t-1}^{B}$ and $\bar{V}_{t-1}^{B}$.

2. we have to show that if the curve 33 intersects the set of feasible values $\left(V_{t}^{B}, V_{t}^{G}\right)$, it cuts the bounds at most twice, so that the set of feasible choices is connected.

To show the first assertion, we look more closely at the family of curves

$$
\phi_{\Delta_{t-1}}: a^{G} \longrightarrow\left[\phi_{B}\left(a^{G}\right), \phi_{G}\left(a^{G}\right)\right]_{\Delta_{t-1}},
$$

where

$$
\begin{aligned}
\phi_{B}\left(a^{G}\right) & =\frac{1}{\beta p_{B}^{\prime}\left(f_{B}^{-1}\left(\Delta_{t-1}+f_{G}\left(a^{G}\right)\right)\right)}, \\
\phi_{G}\left(a^{G}\right) & =\frac{1}{\beta p_{G}^{\prime}\left(a^{G}\right)} .
\end{aligned}
$$


Since $\phi_{G}$ is one-to-one, the curves can also be understood as a function

$$
V_{t}^{B}=\phi_{\Delta_{t-1}}\left(V_{t}^{G}\right) \text {. }
$$

We now want to prove the following: The curves are "decreasing" in $\Delta_{t-1}$, i.e.

$$
\Delta_{t-1}<\Delta_{t-1}^{*} \Rightarrow \phi_{\Delta_{t-1}}\left(V_{t}^{G}\right)>\phi_{\Delta_{t-1}^{*}}\left(V_{t}^{G}\right) .
$$

We do so by calculating the derivative

$\partial_{\Delta_{t-1}}\left(\phi_{\Delta_{t-1}}\right)\left(V^{G}\right)=-\frac{1}{\left(\beta p_{1}^{\prime}\left(a^{B}\right)\right)^{2}} \cdot \beta p_{1}^{\prime \prime}\left(a^{B}\right) \cdot \frac{1}{f_{\theta_{1}}^{\prime}\left(\Delta_{t-1}+f_{\theta_{2}}\left(a^{G}\right)\right)}<0$,

which is negative because of 34 and Condition 2.4. The property of $\phi_{\Delta_{t-1}}$ is reflected by its dotted and the dashed representation in Figure 5.

By the induction hypothesis, the set of $\left(V_{t}^{B}, V_{t}^{G}\right)$ for which $\Gamma_{t}\left(V_{t}^{B}, V_{t}^{G}\right) \neq \emptyset$ is compact and connected. Thus we deduce from 40 that there are $\bar{\Delta}_{t-1}$ and $\Delta_{t-1}$ so that the curves $\phi_{\Delta_{t-1}}$ intersect the set for $\Delta_{t-1} \leq \Delta_{t-1} \leq \bar{\Delta}_{t-1}$ and do not intersect for $\Delta_{t-1}<$ $\Delta_{t-1}$ and $\Delta_{t-1}>\bar{\Delta}_{t-1}$ (of course $\Delta_{t-1}$ could be smaller than zero, the lower limit for $\left.\Delta_{t-1}\right)$. From this ensues the existence of two functions $V_{t-1}^{B}\left(V_{t-1}^{G}\right)$ and $\bar{V}_{t-1}^{B}\left(V_{t-1}^{G}\right)$ limiting the set of feasible pairs $\left(V_{t-1}^{B}, V_{t-1}^{G}\right)$.

To show the second assertion, we have to look more closely at the shape of the curve $\phi_{\Delta_{t-1}}$ as well as the limiting functions $\underline{V}_{t-1}^{B}($.$) and \bar{V}_{t-1}^{B}($.$) . First, we prove that the derivative of \phi_{\Delta_{t-1}}$ is smaller than one. We do so by showing that

$$
D(.) \circ \phi_{G}^{-1}\left(V_{t-1}^{G}\right):=\left(\phi_{G}(.)-\phi_{B}(.)\right) \circ \phi_{G}^{-1}\left(V_{t-1}^{G}\right)
$$

is increasing in $V_{t-1}^{G}$, i.e. the derivative of $\phi_{\Delta_{t-1}}$ is below the one of the diagonal:

$$
\partial_{V_{t-1}^{G}} D\left(\phi_{G}^{-1}\left(V_{t-1}^{G}\right)\right)=1-\partial_{V_{t-1}^{G}} \phi_{B}\left(\phi_{G}^{-1}\left(V_{t-1}^{G}\right)\right)>0 .
$$

Since we know that

$$
\partial_{V_{t-1}^{G}}\left(\phi_{G}^{-1}\right)\left(V_{t-1}^{G}\right)<0
$$

by 25 , it is sufficient to show that

$$
D^{\prime}\left(a^{G}\right)<0 .
$$


Using $a^{B}:=f_{B}^{-1}\left(\Delta_{t-1}+f_{G}\left(a^{G}\right)\right)$ we calculate

$$
\begin{aligned}
D^{\prime}\left(a^{G}\right) & =-\frac{p_{G}^{\prime \prime}\left(a^{G}\right)}{\beta\left(p_{G}^{\prime}\left(a^{G}\right)\right)^{2}}+\frac{p_{B}^{\prime \prime}\left(a^{B}\right)}{\beta\left(p_{B}^{\prime}\left(a^{B}\right)\right)^{2}} \cdot \frac{f_{G}^{\prime}\left(a^{G}\right)}{f_{B}^{\prime}\left(a^{B}\right)} \\
& =-\frac{p_{G}^{\prime \prime}\left(a^{G}\right)}{\beta\left(p_{G}^{\prime}\left(a^{G}\right)\right)^{2}}+\frac{p_{B}^{\prime \prime}\left(a^{B}\right)}{\beta\left(p_{B}^{\prime}\left(a^{B}\right)\right)^{2}} \cdot \frac{p_{G}\left(a^{G}\right) p_{G}^{\prime \prime}\left(a^{G}\right)}{\left(p_{G}^{\prime}\left(a^{G}\right)\right)^{2}} \cdot \frac{\left(p_{B}^{\prime}\left(a^{B}\right)\right)^{2}}{p_{B}\left(a^{B}\right) p_{B}^{\prime \prime}\left(a^{B}\right)} \\
& =\left(\frac{p_{G}\left(a^{G}\right)}{p_{B}\left(a^{B}\right)}-1\right) \cdot \frac{p_{G}^{\prime \prime}\left(a^{G}\right)}{\beta\left(p_{G}^{\prime}\left(a^{G}\right)\right)^{2}} .
\end{aligned}
$$

The last expression is negative by Condition 2.4 and 2.6. Now, the second assertion follows if we can show that the derivative of the boundary functions $\underline{V}^{B}($.$) and \bar{V}^{B}($.$) is greater than one, for$ then $\phi_{\Delta_{t-1}}$ crosses them at most once. So by induction hypothesis, assume that $\underline{V}_{t}^{B}($.$) and \bar{V}_{t}^{B}($.$) have a derivative greater or equal$ than one (note that this is certainly true for the case of $t=T-1$ ).

According to what we have shown above, there are $\bar{\Delta}_{t-1}$ and $\Delta_{t-1}$ that limit the set of values $\Delta_{t-1}=V_{t-1}^{G}-V_{t-1}^{B}$ for which $\phi_{\Delta_{t-1}}$ intersects the set of feasible $\left(V_{t}^{B}, V_{t}^{G}\right)$. From this we might be tempted to deduce immediately both $\underline{V}_{t-1}^{B}$ and $\bar{V}_{t-1}^{B}$ must be linear functions with derivative one, for apparently the limits only depend on the difference $\Delta_{t-1}=V_{t-1}^{G}-V_{t-1}^{B}$. Note, however, that the starting point $\underline{a}^{G}$ (see equation 37) for each curve $\phi_{\Delta_{t-1}}$ is shifting upwards as $V_{t-1}^{G}$ is falling. Thus since by induction hypothesis $\underline{V}_{t}^{B}$ and $\bar{V}_{t}^{B}$ are more steeply increasing than the $\phi_{\Delta_{t-1}}$, we may deduce that

1. indeed $\underline{V}_{t-1}^{B}($.$) is linear with derivative one because the \phi_{\Delta_{t-1}} \mathrm{~s}$ cross the function $\underline{V}_{t}^{B}($.$) at the lower bound \underline{V}_{t}^{G}$ at a high value for $a^{G}$.

2. for lower values of $V_{t-1}^{G}$ the smallest $\Delta_{t-1}$ for which $\phi_{\Delta_{t-1}}$ intersects the set of feasible values $\left(V_{t}^{B}, V_{t}^{G}\right)$ is below the one that would have been obtained with $\underline{a}^{G}$ fixed. Since the latter one would have corresponded to a linear upper bound $\bar{V}_{t}^{B}($.$) with derivative one, we conclude that \bar{V}_{t}^{B}($.$) has to$ rise more steeply than this, i.e. that its derivative is greater than one.

Thus by induction, we have shown that $\phi_{\Delta_{t-1}}$ and $\underline{V}_{t-1}^{B}($.$) and$ $\bar{V}_{t-1}^{B}($.$) cross only once and the second assertion on the form of the$ 
correspondence $\Gamma_{t-1}$ ensues. This concludes the proof of Proposition 3.4.

\section{Proof of Proposition 3.8}

Proof. To prove the proposition, we have to show that at the solution

1. the entitlement constraint 21 of type $G$ is slack,

2. the entitlement constraint 20 of type B is binding,

3. the incentive constraint 19 of type $\mathrm{G}$ is binding.

Beginning with 1 we show that for all contracts $V^{G}>V^{B}$. The assertion then follows by $V^{b, G}>V^{b, B}$ and agent B's entitlement constraint 20 .

So we consider a feasible UI contract. Given any set of effort choices $\left(a_{1}^{B}, a_{2}^{B}, \ldots, a_{T-1}^{B}\right)$ of agent $\mathrm{B}$, the same set of choices would yield a higher value of total expected lifetime utility for agent $G$ than for agent $\mathrm{B}, V^{G}\left(\vec{a}^{B}\right)>V^{B}\left(\vec{a}^{B}\right)$. This is the case because firstly (total) utility when employed is higher than (total) utility when unemployed (compare the remarks 3.5 and .1) and secondly by condition 2.6, first part, $p_{B}\left(a^{B}\right)>p_{G}\left(a^{B}\right)$ for any $a^{B}>0$. Thus, in particular, at the optimum $V^{G}>V^{B}$.

We now prove point 2 by contraction. Suppose that for the solution contracts $\mathrm{b},\left(z_{1}^{b}, \ldots, z_{T}^{b}\right)$, and $\mathrm{g},\left(z_{1}^{g}, \ldots, z_{T}^{g}\right)$, the constraint 20 did not bind. For sufficiently high $\underline{V}$ we may assume that all $z_{t}^{i}>\underline{z}$ for all $t$, in particular for $t=1$. But then create new contracts b' and g' by replacing $z_{1}^{i}$ by $z_{1}^{i}-\epsilon(i=b, g)$ for some $\epsilon>0$ with $z_{1}^{i}-\epsilon>\underline{z}$. These contracts are certainly feasible. They are also incentive compatible, since the entitlements $V_{1}^{i, j}$ are reduced by the same amount. However, the new contracts b' and g' are less costly for the principal, since the cost function $c($.$) is$ strictly increasing. Contradiction.

2.

Point 3 is proved by an argument simular to the one in point 
Figure 2: Set of jointly feasible entitlements in period 11 of 12

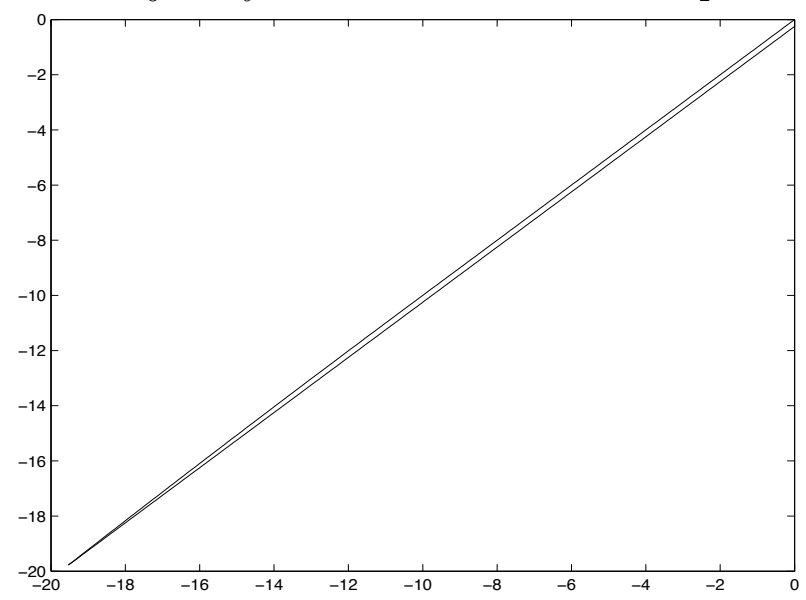

y-axis: entitlement of type $B$, x-axis: entitlement of type $G$

Figure 3: Set of jointly feasible entitlements in period 8 of 12

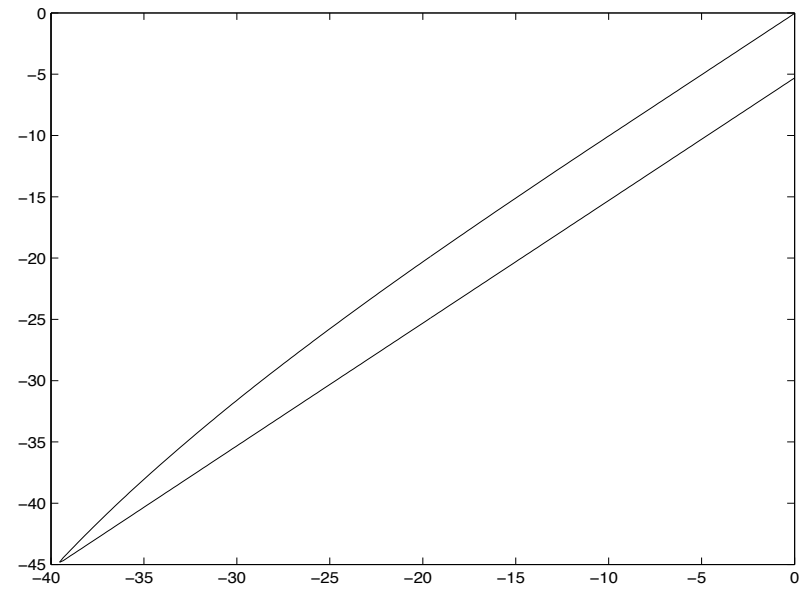

y-axis: entitlement of type $B$, $\mathrm{x}$-axis: entitlement of type $\mathrm{G}$

$\cong$ Certainty Equivalent of $68.67 \%$ of the wage per 
Figure 4: Set of jointly feasible entitlements in period 5 of 12

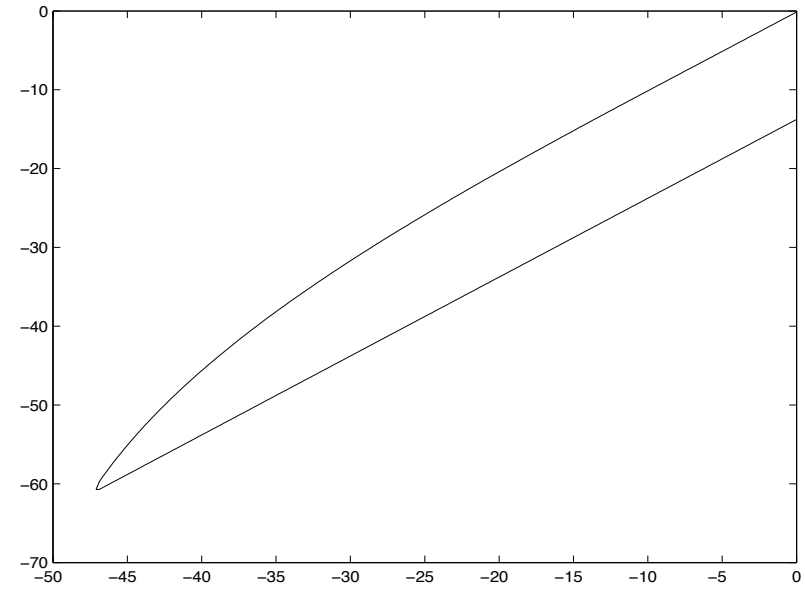

$\mathrm{y}$-axis: entitlement of type $\mathrm{B}$, $\mathrm{x}$-axis: entitlement of type $\mathrm{G}$

Figure 5: Set of jointly feasible entitlements in period 1 of 12

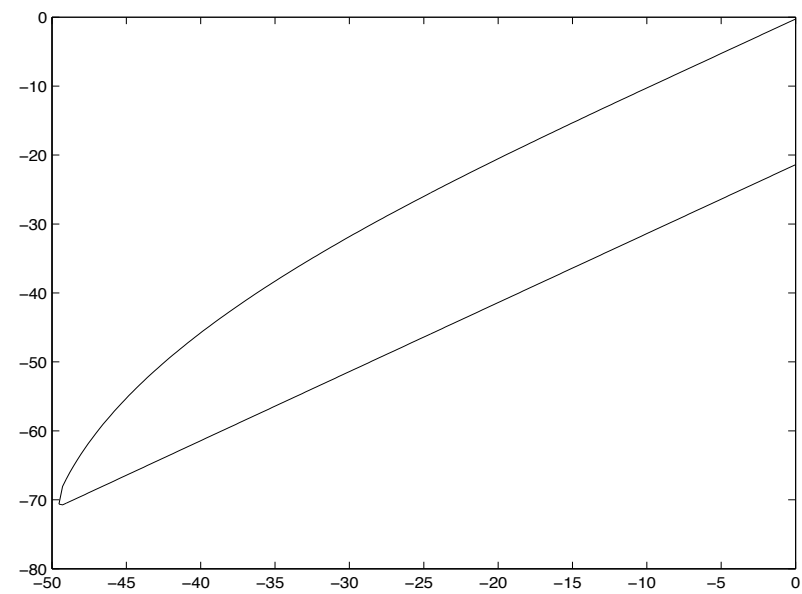

y-axis: entitlement of type $B$, x-axis: entitlement of type $G$ 
Figure 6: UI contracts

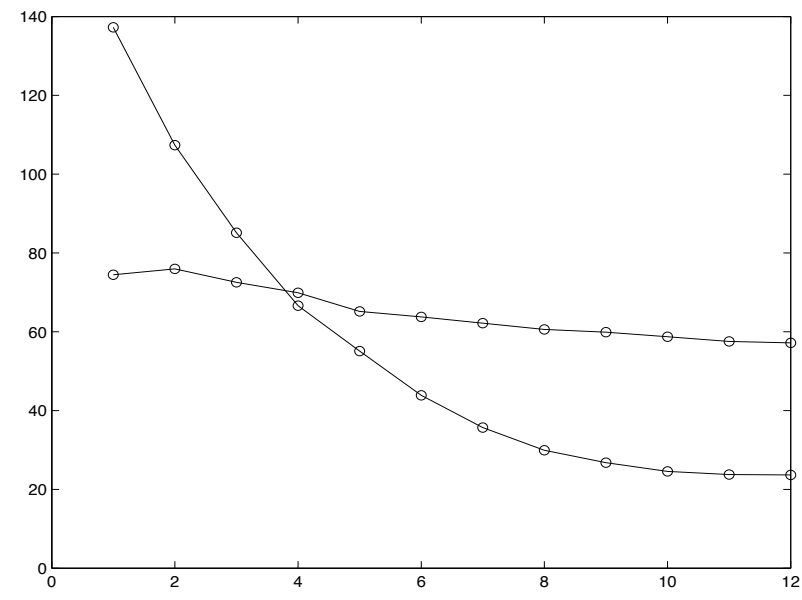

$\mathrm{y}$-axis: replacement rate, $\mathrm{x}$-axis: period

Entitlement bound $\underline{V}=-20$

$\cong$ Certainty Equivalent of $68.67 \%$ of the wage per period

Figure 7: UI contracts

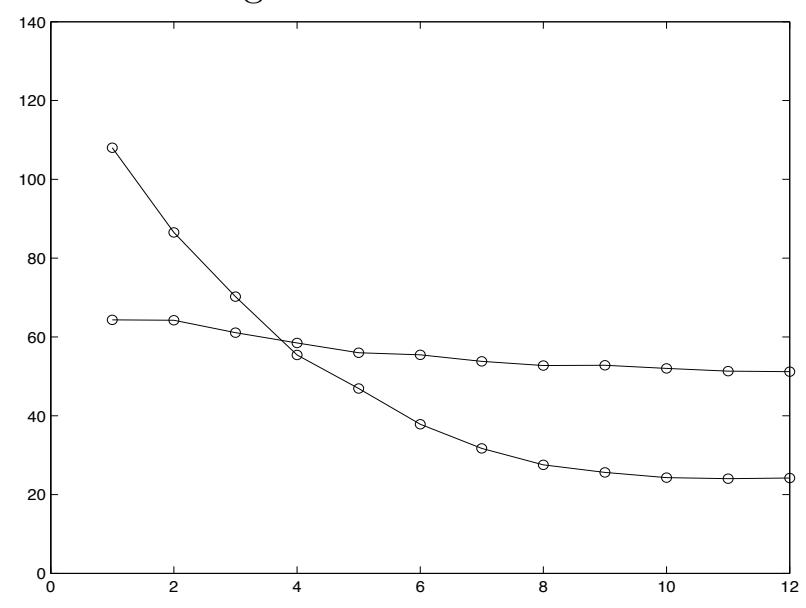

y-axis: replacement rate, $\mathrm{x}$-axis: period

Entitlement bound $\underline{V}=-25$

$\cong$ Certainty Equivalent of $61.76 \%$ of the wage per period 
Figure 8: UI contracts

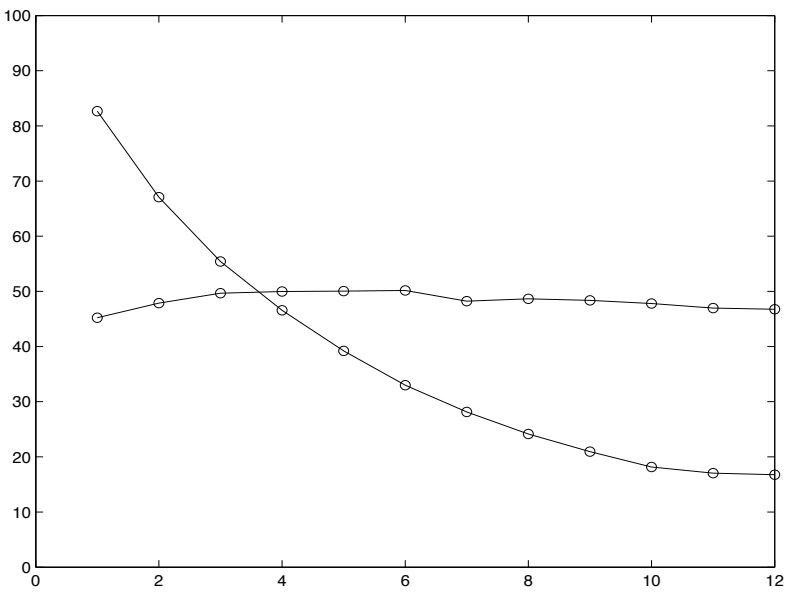

$\mathrm{y}$-axis: replacement rate, $\mathrm{x}$-axis: period

Entitlement bound $\underline{V}=-30$

$\cong$ Certainty Equivalent of $55.21 \%$ of the wage per period

Figure 9: UI contracts

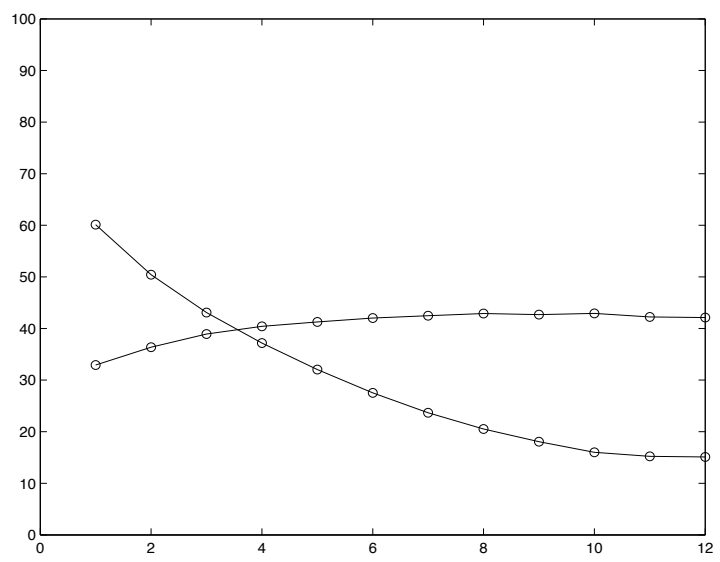

y-axis: replacement rate, $\mathrm{x}$-axis: period

Entitlement bound $\underline{V}=-35$

$\cong$ Certainty Equivalent of $49.03 \%$ of the wage per period 
Figure 10: UI contracts with $q=0.2$

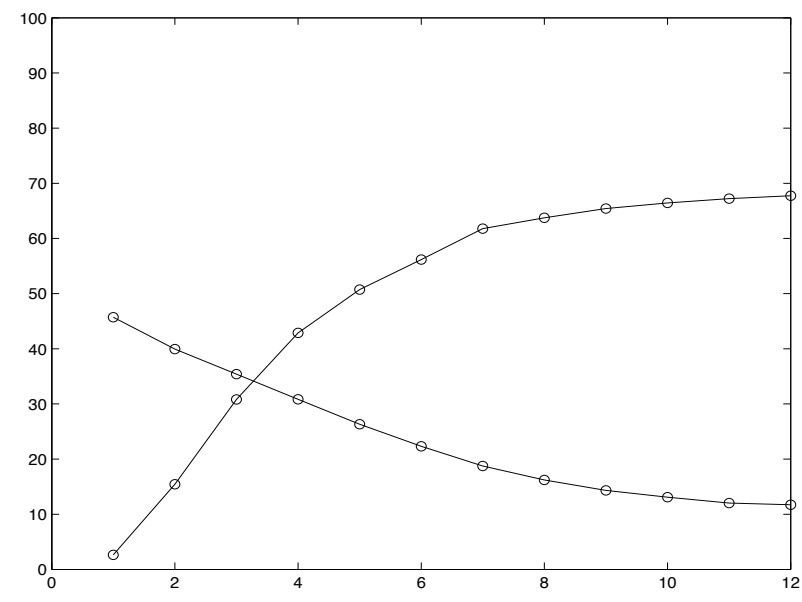

$\mathrm{y}$-axis: replacement rate, $\mathrm{x}$-axis: period

Entitlement bound $\underline{V}=-35$

$\cong$ Certainty Equivalent of $49.03 \%$ of the wage per period

Figure 11: UI contracts with $q=0.8$

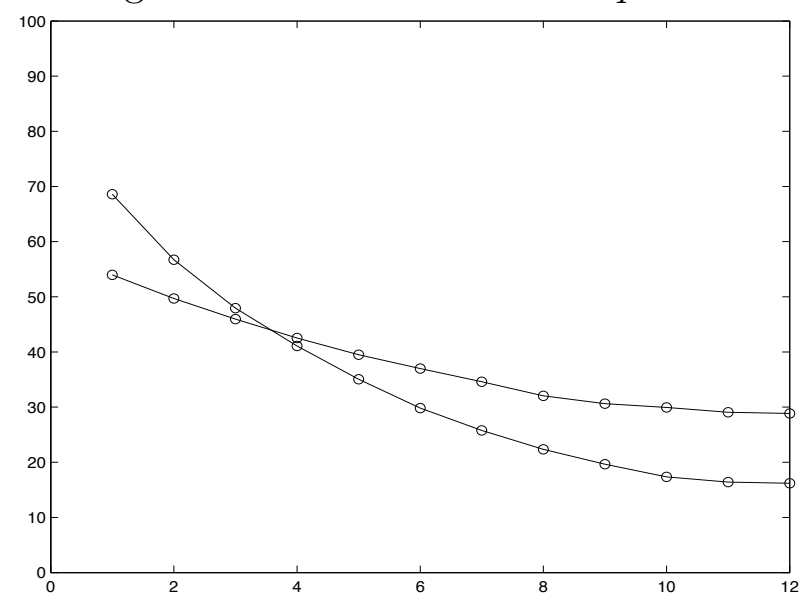

$\mathrm{y}$-axis: replacement rate, $\mathrm{x}$-axis: period

Entitlement bound $\underline{V}=-35$

$\cong$ Certainty Equivalent of $49.03 \%$ of the wage per period 
Figure 12: UI contracts with $\theta_{B}=0.004, \theta_{G}=0.017$

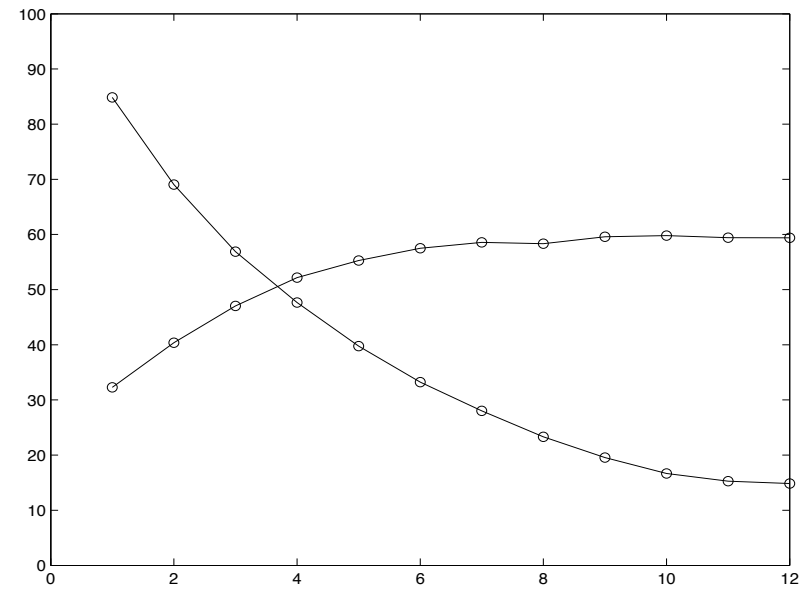

$\mathrm{y}$-axis: replacement rate, $\mathrm{x}$-axis: period

Entitlement bound $\underline{V}=-30$

$\cong$ Certainty Equivalent of $55.21 \%$ of the wage per period

Figure 13: UI contracts with $\theta_{B}=0.010, \theta_{G}=0.017$

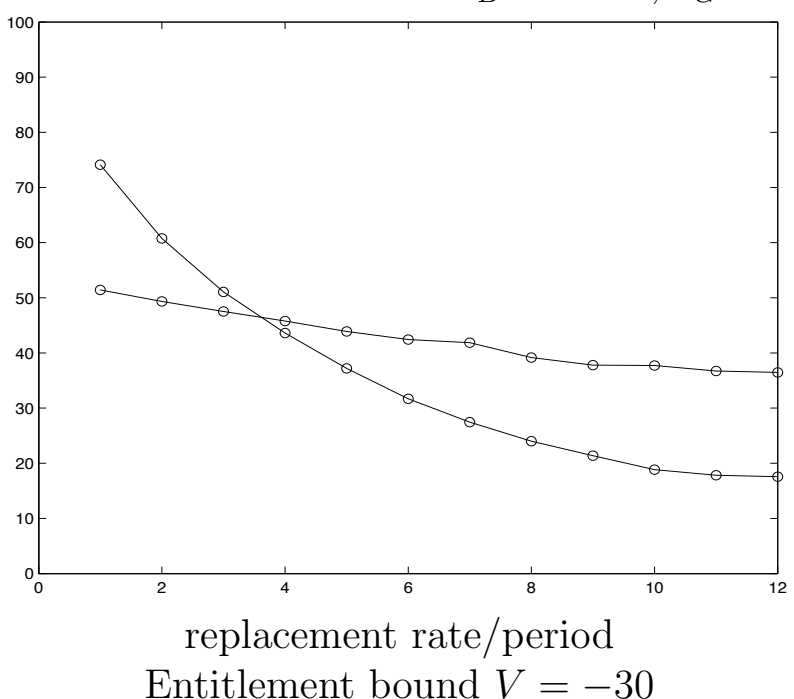

$\cong$ Certainty Equivalent of $55.21 \%$ of the wage per period 
Figure 14: UI contracts with $\alpha=1.5$

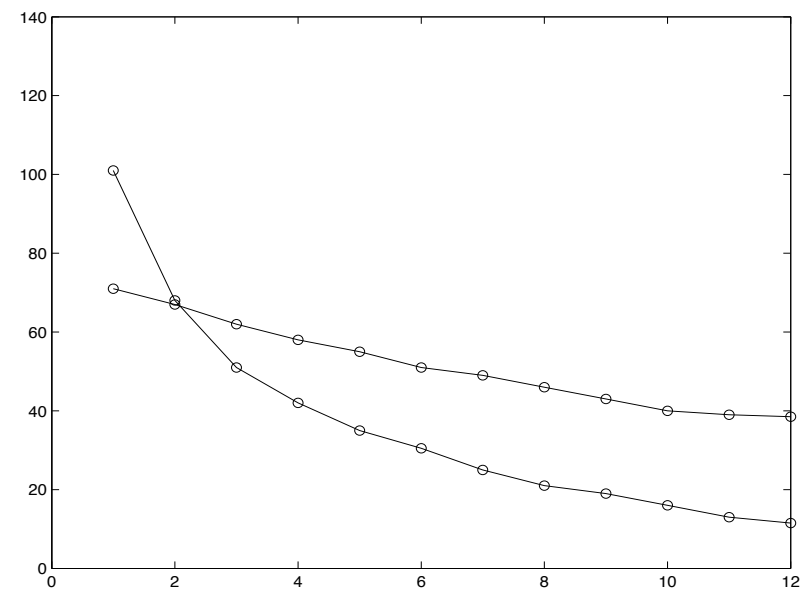

$\mathrm{y}$-axis: replacement rate, $\mathrm{x}$-axis: period

Entitlement bound $\underline{V}=-55.7$

$\cong$ Certainty Equivalent of $68.67 \%$ of the wage per period

Figure 15: UI contracts with $\alpha=2.5$

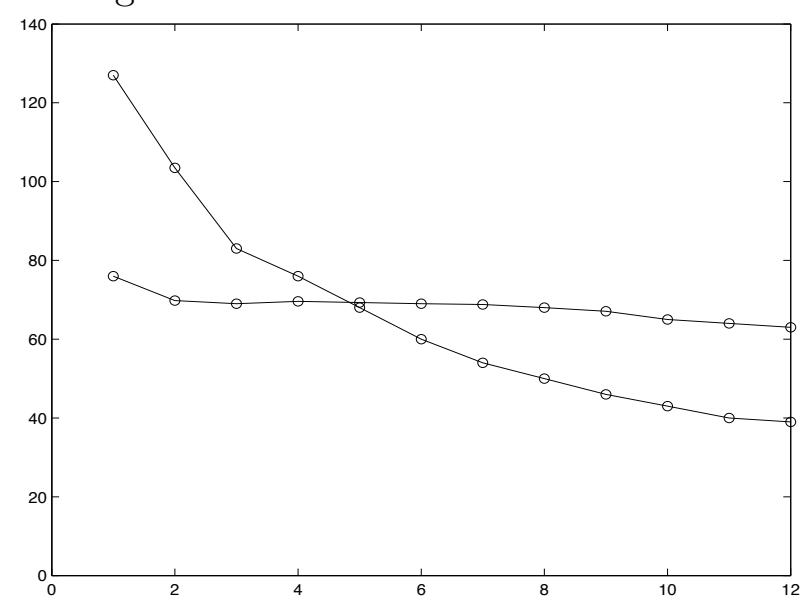

y-axis: replacement rate, $\mathrm{x}$-axis: period

Entitlement bound $\underline{V}=-10.3$

$\cong$ Certainty Equivalent of $68.67 \%$ of the wage per period 
Figure 16: UI contract for two agents, 4 periods

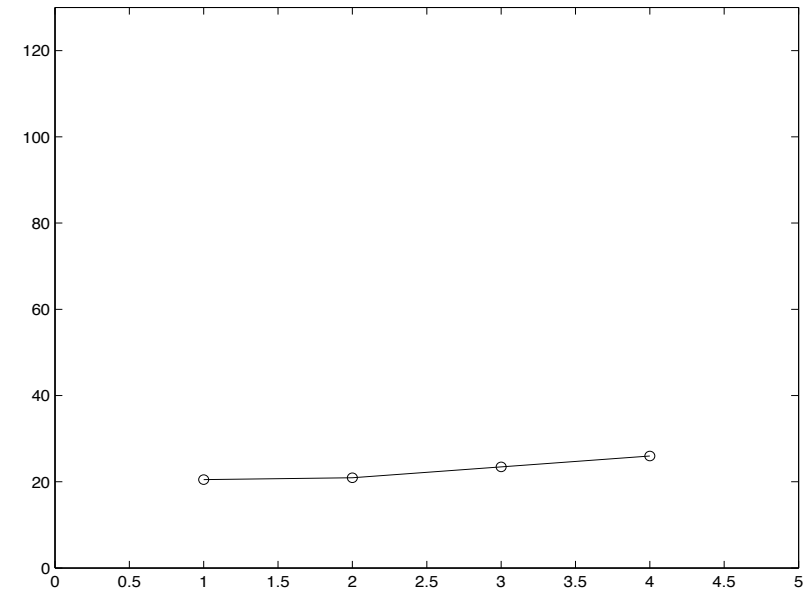

$\mathrm{y}$-axis: replacement rate, $\mathrm{x}$-axis: period

Entitlement bound $\underline{V}=-23$

$\cong$ Certainty Equivalent of $16.60 \%$ of the wage per period

Figure 17: UI contract for two agents, 6 periods

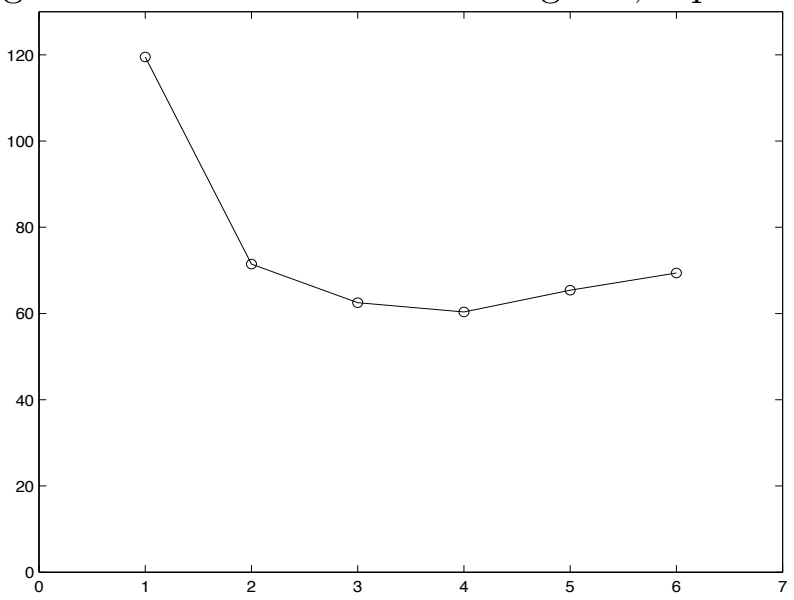

$\mathrm{y}$-axis: replacement rate, $\mathrm{x}$-axis: period

Entitlement bound $V=-10.3$

$\cong$ Certainty Equivalent of $33.17 \%$ of the wage per period 